Portland State University

PDXScholar

Fall 12-28-2018

\title{
Affordances on Facebook, Stress, and Emotional Support
}

Caitlin Rose Rethwish

Portland State University

Follow this and additional works at: https://pdxscholar.library.pdx.edu/open_access_etds

Part of the Social Media Commons

Let us know how access to this document benefits you.

\section{Recommended Citation}

Rethwish, Caitlin Rose, "Affordances on Facebook, Stress, and Emotional Support" (2018). Dissertations and Theses. Paper 4734.

https://doi.org/10.15760/etd.6618

This Thesis is brought to you for free and open access. It has been accepted for inclusion in Dissertations and Theses by an authorized administrator of PDXScholar. Please contact us if we can make this document more accessible: pdxscholar@pdx.edu. 


\section{Affordances on Facebook, Stress, and Emotional Support}

\section{by}

Caitlin Rose Rethwish

A thesis submitted in partial fulfillment of the requirements for the degree of

\section{Master of Science}

in

Communication

Thesis Committee:

Erin Spottswood, Chair

Brian Manata

Cynthia Lou Coleman

\section{Portland State University 2018}




\begin{abstract}
This study discusses Facebook as a social network site and a social media application. It compares perceived emotional support, general life stress, and media affordance-based stress from two participant samples - one that reported using the Facebook desktop site most frequently to reach out for emotional support, and one that reported using the mobile application

The media affordance measure asked participants if perceiving a media affordance was more likely to increase or decrease their stress. In both samples, persistence was more likely to decrease stress, and personalization was more likely to increase stress. On the Facebook desktop site, searchability was more likely to increase stress. On the Facebook mobile application, pervasiveness was more likely to decrease stress, and association to increase stress. When comparing affordances between samples, there were no significant differences found.

When comparing samples, the Facebook mobile application users reported higher life stress, but there was no difference found in perception of emotional support. Within samples, there was no correlation between perceived stress and perceived emotional support.
\end{abstract}

Finally, there was a significant correlation found between perception of emotional support on the site and frequency of reaching out for emotional support. On the Facebook desktop site, users reached out by public post and by private message significantly less frequently if they perceived a higher level of emotional support to be available on the site. On the Facebook mobile application, users reached out by public post significantly 
less frequently if they perceived a higher level of emotional support to be available on the application. No correlation was found for reaching out by private message on the Facebook mobile application. 


\section{Acknowledgements}

I would like to thank my advisor, Dr. Spottswood, for her comments, critiques, engagement, and patience through this entire process. I would also like to thank both members of my committee, Dr. Coleman and Dr. Manata, for their added guidance and input. Finally, I could not have finished this journey without the emotional support and friendship of my research partner, Moira Smith, encouragement and love from my parents, Lisa and Richard Rethwish, and homecooked meals and good humor of my partner, Nathan Mizrachi. 


\section{Table of Contents}

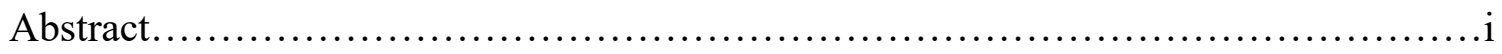

Acknowledgements...........................................................

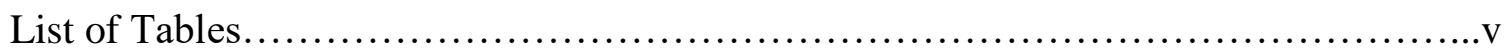

Chapter 1: Introduction.....................................................

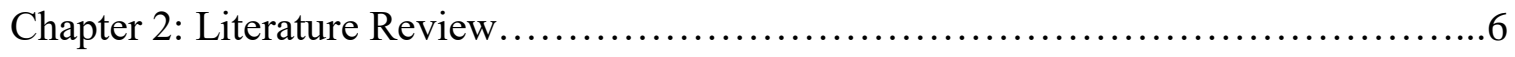

Chapter 3: Methods.....................................................29

Chapter 4: Results.......................................................42

Chapter 5: Discussion..................................................59

References................................................................ 70

Appendices

Appendix A: Informed Consent Form.................................. 77

Appendix B: Use of Communication Channels for Support When Stressed......78

Appendix C: Perceived Stress Scale........................................ 79

Appendix D: Media Affordances Possible on the Facebook Desktop Site........80

Appendix E: Communication Based Emotional Support Scale................83

Appendix F: Demographics..........................................87 


\section{List of Tables}

Table 1 - Media Affordances and Definitions.....................................22

Table 2 - All Results of Paired Samples t-test for Media Affordance Comparisons within the Facebook Desktop Sample.............................................................46

Table 3 - All Results of Paired Samples t-test for Media Affordance Comparisons within the Facebook Desktop Sample ...................................................48

Table 4a - Paired Samples t-test for Media Affordance Comparisons (Higher Means) within the Facebook Desktop Sample...........................................55

Table 4b - Paired Samples t-test for Media Affordance Comparisons (Lower Means) within the Facebook Desktop Sample ..............................................53

Table 5a - Paired Samples t-test for Media Affordance Comparisons (Higher Means) within the Facebook Mobile Sample ..............................................55 Table $5 b$ - Paired Samples t-test for Media Affordance Comparisons (Lower Means) within the Facebook Mobile Sample .............................................56 Table 6 - Independent Samples t-test for Media Affordance Comparisons Between

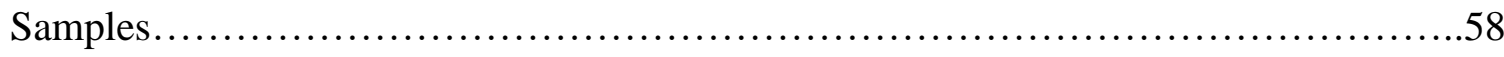




\section{CHAPTER 1: INTRODUCTION}

Since the introduction of the desktop computer into people's homes, researchers have sought to understand how access to the internet and usage of various sites on the internet affect us (Chung, 2014; Coulson, 2005; Gandy-Guedes, Vance, Bridgewater, Montgomery, \& Taylor, 2016; Kraut, Patterson, Lundmark, Kiesler, Mukophadhyay, \& Scherlis, 1998; Shaw \& Gant, 2002). Human processes that have been studied for decades - emotional support, stress, many others - are now being studied in the context of this "new world" online (Gandy-Guedes et al., 2016; Kraut et al., 1998). First, researchers seemed to find that the effects of this new world were entirely negative; increased internet use corresponded with users who were more depressed, lonely, perceived lower emotional support, and exhibited less social connectedness (Kraut et al., 1998). However, over time, the tide has begun to shift. Newer research is finding that we are performing the same social interactions online as we do in person (like exchanging emotional support), and these interactions can affect us in similar - and sometimes positive, sometimes negative - ways (Chung, 2014; Shaw \& Gant, 2002; Shensa, Sidani, Lin, Bowman, \& Primack, 2016).

Social network sites (SNSs), defined as websites where users create profiles, form connections with others, and browse their own and others' connections (boyd ${ }^{1} \&$ Ellison, 2007), are a significant portion of the current internet landscape. According to the Social Media Update for 2016, eight-in-ten online Americans use Facebook (Greenwood, Perrin, \& Duggan, 2016). Social network sites like Facebook are how users connect with their friends, publish relevant information about their lives, and belong to groups that 
represent their interests. Social media applications (SMAs), which are essentially SNSs accessed by mobile phones, are gaining users and popularity year after year as well. While Facebook can be accessed by either desktop site or mobile application, some applications are mostly unusable on a desktop computer. For example, Instagram is meant to be used only by mobile application (desktop users can view the site but cannot make posts), and the same Social Media Update found that $32 \%$ of online adults use Instagram (Greenwood et al., 2016). Researchers have responded by turning to these SNSs and SMAs to research how people are being affected by using this (relatively) new technology. A few common areas of interest are how often people are using them (Shensa, 2016), how users are communicating on them (Coulson, 2005), and how these variables affect users' health (Wright, 2002).

While the above definition for SNSs and SMAs is fairly basic, and the difference will be explored more in-depth in the literature review, it is still clear that there are differences between the two. Researchers have explored how using mobile phones affects a user's health and well-being, and one study found that higher use was correlated with increased feelings of stress (Augner \& Hacker, 2012), so it stands to reason that a website accessed on a mobile phone could affect its user differently than when accessed by desktop computer. However, research rarely attends to these differences. Some research focuses on individual SNSs or SMAs (Liu \& Yu, 2013; Wright, 2002), but the findings become much less relevant when the SNS or SMA changes or disappears. For example, Liu's (2007) article on how social network profiles are a visible performance of interests focuses specifically on MySpace, a social network that is no longer popular, so the 
assumptions, claims, and research questions that focus on features specific to MySpace (i.e. the site's “Top 8" feature) are not applicable to current social network sites. Some research looks at overall usage of many SNSs and SMAs (Shensa et al., 2016), but then the nuances in the differences between the sites and applications is lost. What researchers need is a way to measure how these SNSs and SMAs are affecting users of the technology that attends to what each one offers individually but does not lose its meaning if the SNS or SMA changes or falls out of use. One aim of this paper is to tackle this problem by using the concept of affordances, and Rice, Evans, Pearce, Sivunen, Vitak, and Treem's (2017) conceptualization of media affordances. Media affordances, defined by Rice et al. (2017) are the actions possible on a media based on what the media can do and what the user perceives the media can do, influenced by the user's needs in a certain context. Rice et al. (2017) developed a list of organizational media affordances that fit this definition. By developing and testing this list of media affordances, research can achieve a balance between results that are usefully applied to current media and can still be usefully applied to future media, which is vital in a fast-paced and ever-changing media landscape.

This study also hopes to build on previous research that studies how emotional support and stress are affected by use of SNSs and SMAs, and if perceived emotional support is carried out effectively online. As mentioned earlier, while initial research on internet usage found negative effects on the users (Kraut et al., 1998), current research is showing some positive effects on the users, and a more nuanced view of how SNSs and SMAs are being integrated into users' lives (Chung, 2014; Coulson, 2005; Liu \& Yu, 
2013). While adding to this literature, this study also hopes to acknowledge the differences between the SNS and SMA under study: the Facebook desktop site and the Facebook mobile application.

This paper is part of a larger study done on four different media: the Facebook desktop site, the Facebook mobile application, Instagram, and Snapchat. It also had a fifth version, face-to-face, as a control. Each version of the study was the same, aside from substituting the relevant media where appropriate within the measures. To determine which version participants took, participants were asked to indicate in which way they reached out most frequently for emotional support, and each option corresponded with a version of the survey. This paper will only be discussing, comparing, and analyzing data from two versions of the study: the Facebook desktop site and the Facebook mobile application. Never before explicitly compared in past research, it will be fascinating to discover, within and across these populations: are there significant differences in the users' reports of perceived emotional support? Do users report different levels of overall life stress? Are there significant differences in the users' reports of how their chosen platform for seeking out emotional support affects their stress, based on what the user perceives can be done on that platform? This research will hopefully forge a new path for attending to the differences in SNSs and SMAs and contribute to the media affordance literature.

In Chapter 2, the literature review will explicate the main concepts addressed in the study: perceived emotional support, overall life stress, SNSs and SMAs, and media affordances. In Chapter 3, the methods section will explain how this study 
operationalized each of the concepts covered in the literature review. Chapter 4, the results section, will report on what was found, and Chapter 5, the discussion section, will interpret those findings and review limitations to this study and areas of interest for future research. 


\section{CHAPTER 2: LITERATURE REVIEW}

\section{Emotional Support and Stress}

Emotional support is typically defined within the context of social support. Social support, as laid out by Cobb in 1976, has three parts: the subject believing they are esteemed and valued, that they belong to a network, and that they are loved and cared for. Respectively, those are esteem support, network support, and emotional support. Cutrona and Suhr (1992) later expanded on this definition, adding informational support (seeking and receiving information), and tangible support (seeking and receiving concrete resources, i.e. money), for a total of five components. It's important to first clearly distinguish social support from emotional support because social support is very broad, per the definitions above, and emotional support is more focused.

Emotional support is the act of comforting someone using various communication behaviors, verbal and nonverbal. Emotional support has been defined by Burleson (1984) as helping someone work through being upset by listening, empathizing, and legitimizing their feelings. Cutrona and Suhr (1992) define emotional support similarly, clarifying that the comforter only attempts to support the person without trying to solve the problem, and includes expressions of caring, concern, empathy, and sympathy. The types of topics often breached during emotional support interactions include break-ups, loss of job, divorce, and illness (Burleson, 2003). These difficult life events are often painful and difficult to deal with, and having access to proper emotional support is a vital component to navigating them successfully (Albrecht \& Adelman, 1985). When someone experiencing emotional upset perceives that they have access to resources, regardless of 
the actual utilization of those resources, the increased self-efficacy helps mitigate upset feelings (Cohen \& Wills, 1985). Because of this, measuring perception of access to emotional support is more important than measuring the actual emotional support available - whether or not the person experiencing emotional distress reaches out for support, the perception of having access to the support is what helps the person feel less stressed and more like the problem at hand is manageable.

Online support groups have been studied frequently in the context of emotional support; in 2005, Coulson found that sufferers of irritable bowel syndrome used an online support group as a space to provide emotional support to each other. A decade later, Gandy-Guedes et al. (2016) studied another online support group for social workers and found that the members used the space to exchange emotional support and informational support. Even blogs have been shown to help people feel as though emotional support has been accrued through sharing personal stories and making new connections, in a pseudosupport group environment (Chung, 2014).

To measure perceptions of communication-based emotional support, Weber and Patterson (1996) developed the Communication Based Emotional Support Scale (CBESS). The researchers developed the scale to measure perceptions of emotional support from romantic partners, and CBESS is defined as being directly concerned with support as a product of interpersonal relationships. Although it was originally intended to be used to measure perceived emotional support from romantic partners, it has been successfully applied to many different contexts, including online support groups. In 2002, Wright applied CBESS to measure cancer patients' perception of emotional support in 
online groups, and found that when the patients perceived higher levels of emotional support in the online group, it was correlated with slightly lower reports of perceived life stress. Wright applied this same scale again in 2012, this time to Facebook. Facebook users were asked to report on their perception of emotional support from their network on Facebook, and also their perceived life stress. The results showed that when participants perceived a higher level of emotional support, it was correlated with lower reports of life stress. Clearly, emotional support is extremely significant in close relationships, and receiving good emotional support is not only an indicator of well-being (Burleson, 2003), but also increases self-efficacy to tackle the problems causing stress (Bolger, Zuckerman, \& Kessler, 2000).

While emotional support is fairly well-defined in the literature, aside from its conflation with social support, there are multiple ways to conceptualize and operationalize stress. The broad characterizations of stress fall into three categories: environmental, which focuses on specific events that are deemed stressors; psychological, which focuses on the participants subjectively assessing their own stress; and biological, which focuses on the body's physical reactions to stressors (Cohen, Kessler, \& Gordon, 1997). After acknowledging that there are multiple conceptualizations and measurements of stress, Cohen et al. (1997) attempt to provide a definition that can be used across the board: stress is "a process in which environmental demands tax or exceed the adaptive capacity of an organism, resulting in psychological and biological changes that may place persons at risk for disease," (p. 3). This combines the environmental, psychological, and 
biological perspectives into one conceptualization that can be used to ground all three disciplines.

The psychological perspective of stress, which deals with participants assessing their own stress (Cohen et al., 1997), is frequently used in communication research (Guan, Chiang, Sherman, Nguyen, Tsui, \& Robles, 2017; Welbourne, Blanchard, \& Wadsworth, 2013; Wright, 2002; Wright, 2012). While stress brought on by specific events comes and goes, overall psychological stress ascertains whether a person's stress over time is higher or lower. To assess overall psychological stress, Cohen, Kamarch, and Mermelstein (1983) developed the Perceived Stress Scale (PSS). The PSS aims to measure the degree to which participants perceive their life has been volatile or overwhelming over the previous month (Cohen et al., 1983).

Having proper emotional support is a vital component to navigating these difficult events successfully (Albrecht \& Adelman, 1985), and lowering feelings of stress (McKinley, 2013). The buffering model of support claims that during these times of increased stress, if someone perceives that social support is accessible, he or she is likely to be less stressed (Cohen \& Wills, 1985). Therefore, if you compare one person who consistently perceives that social support is available when stressful life events occur, that person's general life stress should be lower compared to another person who encounters stressful events and perceives that less social support is available - this availability of social support will buffer the first person from feeling stressed, while the lack of social support will mean that the second person is not buffered from the stress. According to the authors of the buffering model, this model is supported when the study 
measures the perceived availability of coping resources - not the actual availability, or the actual utilization (Cohen \& Wills, 1985). The most important component is the belief that the person experiencing the stress has access to resources, regardless of deciding to use them (Cohen \& Wills, 1985). The perceived support network acts as a buffer from the stress, and the person experiencing the difficult life event perceives that her network is there to help her better cope with her problems if she needs it, which mitigates her stress.

One of the critiques of the buffering model of support is that when the buffering effects of support are measured, support is not properly conceptualized or operationalized (Thoits, 1982). As stated in the beginning of this section, social support is a broad term, and has five components: informational, esteem, tangible, social network, and emotional support. In the original conception of the buffering model of support, support is only indirectly defined as access to a network, psychological resources, and the existence of social relationships (Cohen \& Wills, 1985). This roughly corresponds with network support and emotional support, but is not well conceptualized, and seems to rest on measures of actual support instead of perceived support. This is a limitation to the original model, and caused inconsistencies in early applications of the model when it came to measuring support - for example, some researchers simply defined support as the existence of a spouse and living with others (Eaton, 1978; Myers, Lindenthal, \& Pepper, 1975), while Kaplan, Cassel, and Gore (1977) defined support only as "the relative presence or absence of psychosocial support resources from significant others," (p. 50). More recent applications have tended to use scales that attempt to measure perceived social support, for example, by focusing on various sources that a participant perceives 
support to be available from (i.e. spouse or friends) (Graham \& Barnow, 2013), or if participants feel that the quality and quantity of the support received from their network is high (Beckley, 2006). However, this still does not address the murkiness of social support as a concept. Separating out social support into its various components and measuring how each component works to buffer someone from stress will lend clarity and focus to the buffering model. This study will focus on emotional support specifically, although each component of social support should be considered for future research.

This leads to a set of research questions on emotional support and stress. This research, as mentioned in the introduction, is being done on two separate populations, one of which uses the Facebook mobile application most frequently for emotional support, and the other of which uses the Facebook desktop site most frequently for emotional support. These research questions seek to explore if there are any statistically significant differences between the two populations when it comes to perceived emotional support and perceived life stress. If there are differences, it could support literature that has found that using a mobile phone affects users differently than a desktop site (Augner \& Hacker, 2012); if there are no differences, it could suggest that there are other factors at play aside from device usage. Either way, researching the differences is an important addition to the literature. The research questions are as follows:

RQ1: When looking at participants' personal judgment of general life stress, which group is more likely to report greater stress: users of the Facebook desktop site or users of the Facebook mobile application? 
RQ2: When looking at participants' personal judgment of perceived emotional support, which group is more likely to report higher perceptions of emotional support: users of the Facebook desktop site or users of the Facebook mobile application?

RQ3a: Is there a statistically significant relationship between perceived stress and perceived emotional support available when participants indicate using the Facebook desktop site most frequently for emotional support?

RQ3b: Is there a statistically significant relationship between perceived stress and perceived emotional support available when participants indicate using the Facebook mobile application most frequently for emotional support?

Because of the buffering benefits of perceived emotional support on stress, researchers are clearly drawn to measuring the relationship in various ways, including if and when the buffering effects exist when the support is received online. The relationship between stress and support has been studied in online support groups (Meier, 2002), and researchers are also moving to SNSs and SMAs, such as Facebook (Gandy-Guedes et al., 2016; Greene, Choudhry, Kilabuk, \& Shrank, 2011). The following section will give a brief history and overview of SNSs and SMAs, along with existing literature on emotional support and stress on SNSs and SMAs.

\section{Social Network Sites, Social Media Applications, and Frequency of Use}

Social network sites (SNSs) are traditionally thought of as websites where users create profiles, form connections with others, and browse their own and others' connections (boyd \& Ellison, 2007). The communication that happens on these sites has been referred to as the Web 2.0 (Kaplan \& Haenlein, 2010), because it is unique from 
what communication was allowed when the web first launched. On SNSs, and for the first time on the web, you could "articulate and make visible" your social network (Ellison \& boyd, 2007, p. 211). On Facebook, this public articulation of your social network is referred to as a user's "friend list." What this means is that a user has a profile that is unique to him, and other users of the site can visit his unique profile and see which other users he has agreed to connect with. On Facebook, some users implement privacy settings that make this public articulation of the user's friend list private, so that only other connected users can view the friend list. When these types of sites first arose, they were more focused on network growth possibilities, hence the characterization of the sites as "network" sites. Users prioritized connecting with as many other users as possible. More recently, the sites have evolved to focus more on creating content and viewing and interacting with other users' content, both within the user's articulated social network and outside it. In other words, instead of creating a large network of mutual connections, users seem to prioritize creating and posting the user's own content as well as interacting with other users and commenting on other users' posts on these sites (Ellison \& boyd, 2013). Because of this, Ellison and boyd (2013) amended their definition of SNSs to include an additional component: that participants "can consume, produce, and/or interact with streams of user-generated content provided by their connections on the site," (p. 7). This marks a huge shift in how we use SNSs, and this new focus also carries over to social media applications (SMAs).

Social Media Applications are similar to, and often affiliated with, popular SNSs, but SMAs have distinct differences that likely affect how they are perceived and used. 
For example, SNSs are typically accessed on a desktop computer whereas SMAs are typically accessed on a mobile phone. This shift from a desktop site to a mobile application affects the functionality of how a user can interact with other users, even if it is through the same parent company's "platform." For example, on the Facebook desktop site (an SNS), the messaging function is fully integrated into the site. When visiting any page on the site, a user can simultaneously view the content on the page (for example, content on a group page) and a list of connected friends on the right side of the page. The user can select any friend on this list, and a small box will appear on the current page. This smaller box is where the user can send the chosen friend a message, and even have a full conversation, without needing to leave the open page. The Facebook mobile application (an SMA) has no integrated messaging feature, and the user must exit the main application and open a separate Messenger application in order to send and receive messages from their Facebook contacts or friends. While the Facebook mobile application is widely used, surpassing 1.3 billion active monthly users as of September, 2017 (Constine, 2017), this lack of integration could discourage a mobile application user from attempting to obtain emotional support via private message from a friend, because the user may not want to navigate away from the main application. Due to the lack of research in this area, we do not know if users perceive these differences in functionality to be important, or if it affects how the user chooses to use the site or the application to seek out emotional support.

As researchers became interested in studying how the usage of SNSs and SMAs was affecting users of the technology, usage had to be conceptualized. One of the most 
well-known initial studies on how internet use affected the users was done by Kraut et al. in 1998. The researchers gave a computer and software to the families that agreed to participate, and the computers came installed with software that logged internet use - this meant an unusual advantage of being able to know exactly how many hours, down to the minute, that the participants spent online. This study was interested in how internet usage affected social involvement and psychological well-being, which was measured with pretests and post-tests. The researchers found that higher internet usage was correlated with lower social involvement and decreased psychological well-being, and this set the tone for research done on how internet usage affects those using it for the decades to come.

However, not all researchers were able to give their participants computers and get accurately logged hours spent on the internet, and so measures of internet usage have been largely self-reported. For Facebook studies in particular, Ellison, Steinfield, and Lampe (2007) developed the Facebook intensity scale, which measures how intense of a presence Facebook is in participant's lives. This scale includes a question asking how many minutes per day the participants spent on Facebook in the previous week. They found that a higher intensity score was positively correlated with higher maintenance and creation of social capital (Ellison et al., 2007). This scale was adapted by Liu and Yu in 2013, and found similar results: higher Facebook intensity predicted higher perception online social support.

While measuring the intensity of Facebook in participants lives has been an effective tool, other researchers have been interested in how usage of multiple SNSs and SMAs is affecting the users, and therefore tend to use a more simplified conception of 
usage. Shensa et al. (2016) studied how the usage of 11 popular social media platforms affected perceived emotional support, and measured this by having participants indicate how much time they spent on each platform each day, as well as how many times per day they visited each platform. Shensa et al. (2016) found that those who reported higher usage of all social media platforms were more likely to report lower perceived emotional support. Finally, in Rice et al.'s (2017) study on organizational media affordances, the researchers developed a measure that asked participants to indicate how frequently they used each type of organizational media communication (including conferencing, texting, and WhatsApp), on a scale that ranged from never to many times per day. Rice et al. (2017) tested the usage data of each media for correlations with each of the media affordances - for example, they found that higher levels of texting were positively correlated with all of the affordances in their scale (visibility, editability, selfpresentation, awareness, pervasiveness, and searchability).

There have been studies done attempting to find relationships between emotional support and SNS use, but the measure of usage is not always useful in coming to conclusions about the SNSs and SMAs the participants are using. As mentioned, Shensa et al.'s (2016) study looked into perceived emotional support and SNS use; however, while the measure asked for usage of 11 different SNSs, the results combined the responses into a single item. This adds to the literature that is concerned with how overall use of media affects its users, but cannot be parsed out into individual media (which are very likely to be having different impacts on the user). In the same vein, the Facebook Intensity Scale (Ellison et al., 2007) not only specifically pertains to Facebook, but usage 
and frequency is only one item within the scale. This is an excellent scale to get a better sense of how Facebook is affecting the user on a deeper, overall level; scale items include, "I am proud to tell people I am on Facebook," and "I feel I am part of the Facebook community" (Ellison et al., 2007, p. 1150). However, as an 8-item scale, it becomes more difficult to apply to many media within one survey. Utilizing a usage measure that takes into account the various SNSs and SMAs individually, and incorporating a measure that addresses effects of that usage on the user (the affordance measure, discussed later in the paper) provides a result more grounded in the aims of the study. This study will adapt a measure from Rice et al. (2017) to look at how often participants are using Facebook to reach out for emotional support; using media affordances as a lens will allow this study to address how the perception of the site is affecting the user.

Shensa et al.'s (2016) study is also problematic in terms of determining how perceptions of these technologies affect their use as emotional support tools. The study 1) did not reveal if the exchange of emotional support is possible on any of the sites individually, 2) the various SNSs under study are all very different (Facebook is a content-focused social media, while Vine and YouTube are primarily used to view video content), and users likely have different perceptions of how these technologies can, and should, be used, and 3) those perceptions will greatly influence if, when, and how a user would perceive any of these SNSs and/or SMAs as effective tools to exchange emotional support. 
In fact, there are studies that indicate users can perceive SNSs and SMAs as effective tools to offer and/or obtain emotional support. Wright (2012) focused on college students using Facebook, asking the participants to report on their perceived emotional support from whoever they felt provided them the most support on Facebook, and overall life stress. The study revealed that when users indicated greater perceptions of emotional support from Facebook partners, it was correlated with lower overall stress. Focusing on communication behaviors on Facebook, instead of a general measure of overall Facebook use, can reveal whether a specific action is possible on the site - like the exchange of emotional support. This leads to a set of research questions related to frequency of use and emotional support:

RQ4a: Is there a relationship between perceived emotional support and frequency of using the Facebook desktop site to reach out for emotional support?

RQ4b: Is there a relationship between perceived emotional support and frequency of using the Facebook mobile application to reach out for emotional support?

In addition to focusing on specificity of the behavior on the SNS or SMA, more clarity is needed for whether the researchers are referring to the desktop site or mobile application. In Wright's (2012) study, it is unclear whether participants were referring to perceived emotional support on Facebook's desktop site or mobile application, and considering the functional differences between the two, participants may have different perceptions of how the two can and should be used. This difference could appear in how the users view the technologies in general, as well as the users' perception of the technology for the exchange of emotional support specifically. The lack of research in 
this area means that there is no literature that provides support for finding a difference in perception of the site and the application. However, the differences in how the site and application are accessed, as well as the differences in functionality, provide enough justification to begin exploration into this area. If a user does indeed perceive enough of a difference between the Facebook site and Facebook mobile application that it affects his stress, it may affect his usage of the site or application for emotional support. As usage of these communication technologies saturates our lives, understanding how we communicate on them is more important than ever. Whether differences are found in this case or not, it is an important contribution to this fresh and ever-changing area of research, and even if no differences are found, this is an area that should continue to be explored. Of course, measuring how users perceive these communication technologies is not easy. These different perceptions are more clearly understood through the lens of media affordances.

The following section will define and describe what affordances and media affordances are, and how media affordances can affect stress when it comes to using an SNS versus an SMA. It will also explain how we can understand, through the lens of media affordances, why past findings examining emotional support on SNSs and SMAs have been found to conflict with each other, and finally, which media affordances could be relevant for those seeking to obtain emotional support by using an SNS or an SMA.

\section{Media Affordances and Stress on Facebook}

Affordances. Gibson (1979) was one of the first to define and apply the concept of affordances to social psychology phenomena. His definition of affordances was action- 
based, asserting that affordances were the possibility of an action, whether or not the actor perceived the action to be possible. Under this definition, the affordance lived within the object, and while the user could perceive the affordance, the affordance existed independently of the user's perception and experience. In contrast, Norman (2013) attempted to redefine affordances as more of a relationship between the physical properties of an object with the needs and wants of the person who sees that object. Under this definition, the affordance lives not within the object, but somewhere between the object and what it is capable of being used for, and the person who sees that object as a way to satisfy a particular need or desire, in accordance with their own needs, culture, and understanding of the object (Norman, 2013). The difference between the two definitions seems to be in perception, as Gibson's (1979) definition addresses actual affordances, while Norman's (2013) definition points more towards perceived affordances. When measuring users' perceptions of the site under study, Norman's (2013) conceptualization is clearly the better fit.

Affordances are not features, but features make affordances possible. For example, a large flat rock affords sitting, and a tired hiker will likely perceive it to afford sitting. The rock likely has the features of being large and flat on top, but it does not afford being large and flat - its size and shape afford sitting. This perceived affordance of sitting is also influenced by the needs and perception of the user. The hiker has a need to sit down somewhere to rest, and she understands that the rock affords that. If the hiker does not have a need of resting, she will likely pass the rock by without perceiving it to afford sitting. However, whether or not she perceives it, the rock still affords sitting. 
Media Affordances. This concept of affordances has gained more popularity amongst those researching communication technology because it can help researchers focus on the possible actions and the users performing them, rather than focus on technologies that may be popular now but may become obsolete in the future (Ellison \& Vitak, 2015; Evans, Pearce, Vitak, \& Treem, 2016; Rice et al., 2017). When affordances are applied to information and communication technologies (ICTs), the ICT becomes the object, and affordances become media affordances (Rice et al., 2017). A media affordance is a user's perception of the ways that ICT - like the Facebook desktop site can be used. This perception of the media affordance is influenced by what the he needs, how he's seen others use the object, and how he perceives the object can be used according to social norms (Ellison \&Vitak, 2015; Evans et al., 2016; Gibson, 1979; Norman, 2013; Rice et al., 2017). Media affordances are affordances possible on ICTs specifically, and can be applied to many different ICTs.

Rice et al. (2017) propose a definition of media affordances: "relationships among action possibilities to which agents perceive they could apply a medium (or multiple media), within its potential features/capabilities/constraints, relative to the agent's needs or purposes, within a given context." (p. 109). This aligns more closely to how Norman (2013) conceptualized affordances, because the affordance relies partially on the perception and needs of the agent. Rice et al. (2017), applying the concept to organizations, developed a list of organizational media affordances. However, these organizational media affordances are not solely applicable to organizational communication. The three central assumptions that the researchers make about 
organizational media affordances are that the affordances 1) are perceived by agents as available within the organizational context, 2) can be associated with one or more groups of organizational media, and 3) occur on an interpersonal, group, and organizational level (Rice et al., 2017).

While these organizational media affordances were originally conceptualized for organizational communication, the concept of media affordances can be easily applied to multiple media contexts. Each of the media affordances can be found on various types of media, including Facebook. The list of media affordances that Rice et al. (2017) developed, along with their definitions, can be found in Table 1 below.

\begin{tabular}{|l|l|}
\hline Table 1: Media Affordances and Definitions \\
\hline Association & $\begin{array}{l}\text { The ability to establish connections between individuals, } \\
\text { between individuals and content, or between an actor and a } \\
\text { presentation }\end{array}$ \\
\hline Awareness & $\begin{array}{l}\text { Ability to be aware of the information others on the network } \\
\text { have; be aware of activities, opinions, or locations of others } \\
\text { that use the network; keep up-to-date with other users' } \\
\text { discussions; keep up-to-date with network policies/norms }\end{array}$ \\
\hline Editability & $\begin{array}{l}\text { Ability to spend a good deal of time and effort crafting and re- } \\
\text { crafting a post before it is viewed by or accessible to others }\end{array}$ \\
\hline Persistence & $\begin{array}{l}\text { Ability to make content accessible in the same form as the } \\
\text { original display after the user has posted/shared content }\end{array}$ \\
\hline Personalization & $\begin{array}{l}\text { Ability to include information, photos, and other content on } \\
\text { social media that is representative of a user's personal } \\
\text { identity; adjust social media presence to their preferences }\end{array}$ \\
\hline Pervasiveness & $\begin{array}{l}\text { Ability to get responses to requests from other users; } \\
\text { communicate with other users while moving, commuting, } \\
\text { traveling }\end{array}$ \\
\hline Searchability & Ability to search for content posted by self or others \\
\hline Sharing & $\begin{array}{l}\text { Ability to create groups for sharing information about specific } \\
\text { topics, needs, or concerns; obtain and use others' posts, } \\
\text { photos, other information; share thoughts/feelings, photos, } \\
\text { physical location, other information with other users }\end{array}$ \\
\hline Evaluability & $\begin{array}{l}\text { Ability to evaluate other users' information by providing my } \\
\text { recommendations, comments, liking, or tagging; see other }\end{array}$ \\
\hline
\end{tabular}




\begin{tabular}{|l|l|}
\hline & $\begin{array}{l}\text { people's evaluation of information through their } \\
\text { recommendations, comments, liking, or tagging, following }\end{array}$ \\
\hline Visibility & $\begin{array}{l}\text { Ability to make behaviors, knowledge, preferences, physical } \\
\text { location, and communication network connections that were } \\
\text { once invisible (or at least very hard to see) visible to other } \\
\text { users in the network }\end{array}$ \\
\hline Signaling & $\begin{array}{l}\text { Ability to receive notifications about other people's } \\
\text { information or updates }\end{array}$ \\
\hline
\end{tabular}

Stress. Rice et al.'s (2017) conception of media affordances is very new research, and thus not yet widely applied. However, there have been a handful of studies published in the last year that use this concept of media affordances, and some attempt to at least tangentially link media affordances with stress. The most relevant example for this paper is a study done by Afifi, Zamanzadeh, Harrison, and Callejas (2018), wherein the researchers took a biological approach to stress and studied the relationship between technology use, affordances, and stress. The researchers first link high usage of technology with greater impact of the affordances of that technology, and posit that each affordance has the potential to be a beneficial or stressful aspect of using the technology. For example, the heightened connectivity that Facebook brings, and therefore greater visibility (one of the media affordances defined above), can bring on heightened stress. The researchers measured stress by taking saliva samples and testing for cortisol levels, a classic stress marker (Afifi et al., 2018). The results showed greater levels of stress in adolescents than in their parents, finding that adolescents had higher usage of social media and higher levels of stress, but no relationship between email usage and stress; their parents, on the other hand, displayed no relationship between social media usage and stress, but did have greater stress associated with greater email usage. Unfortunately, 
the researchers did not measure how the media affordances affected participant stress directly, only measuring technology use and stress, and using the concept of media affordances to interpret their results.

The current study is interested in each media affordance individually, which will lend a greater understanding of how different technologies with different mixes of affordances can affect users of those technologies. However, instead of being interested in whether participants perceive these media affordances to be available or not, this research is concerned with whether perceiving the media affordances to be available affects the user's stress when using the platform. Measuring whether or not participants perceive the affordance to be available will only provide a list of perceived affordances of the media under study. Grounding the measure in how perceiving the affordance affects the user's stress will provide insight into how users are affected by the media they use.

Media Affordances on Facebook. The original intent of the list of media affordances from Rice et al. (2017) remains, which is to be a list of media affordances that exist on and can be applied to multiple different media, as opposed to being created based around one media. This list can be very helpful to get a sense of how an SNS or SMAs can be used. For example, Facebook has a search bar that users can utilize to search for any person, event, group, or topic. Facebook's search bar feature, therefore, affords searchability. If Facebook is perceived as affording a user with the ability to search for specific people or kinds of content (i.e., searchability), it means that a user can search for and find people to reach out to for emotional support or search the site to find posts about how to deal with specific issues or sensitive matters. As such, searchability 
may help a user get emotional support if the user perceives he can search for people or content on an SNS or SMA that can help mitigate or alleviate his emotional upset.

However, there is more to the media affordance puzzle than mere perception. Understanding which affordances are perceived on which media is useful, but only when also determining how different media affordances affect that media's users. For this study, the focus is on stress, to tie in with the earlier part of the study on perceived stress. The argument for the link between emotional support and stress is that when a person perceives that he has access to emotional support, he can be buffered from the effects of stressful life events. It's also been established that people are turning to SNSs and SMAs to obtain and exchange emotional support. However, we do not know how media affordances are affecting a user's stress. A study done on perceived affordances on an SNS or SMA would give us insight into how users think an SNS or SMA can be used, but not how it is affecting the users themselves. This study is grounded in perceived emotional support and stress, so one of the aims is to discover if the various media affordances are also affecting user stress positively or negatively, as this could affect whether or not they choose to utilize the site to obtain emotional support. The example was used earlier that a user could utilize the searchability that Facebook affords to search for a support group to obtain emotional support. However, if a user perceives that a post they make on her profile can be searched for by other users - for example, a post about emotional struggles and stress - she may feel stress due to worrying how those other users will interpret her post. When thinking about publishing a public post asking for emotional support, she could also imagine a future employer searching for her profile on Facebook, 
and that could cause her stress. In this way, the affordance of searchability may actually increase her stress and discourage her from posting about her problems on an SNS or SMA. Reaching out for emotional support is supposed to help reduce stress (McKinley, 2013), but if the SNS or SMA has affordances that increases a person's stress, these perceptions will hinder the site or application's perceived viability as place to reach out and get emotional support.

In addition, if a user perceives that an SNS affords them something that an SMA does not, it will affect which one is chosen to use to reach out for emotional support. The current literature ignores the differences in affordances between SNSs and SMAs, and most studies either generalize to all social media (Shensa et al., 2016), or focus on Facebook generally without distinguishing between the desktop site or mobile application (Wright, 2012). These oversights muddle our ability to understand how using an SNS or SMA could be stressful, especially when it comes to using either/or to obtain emotional support. As previously addressed, there are some differences between the features, and thus potentially between the subsequent affordances, of the desktop and mobile iterations of Facebook. One major difference is how the two are accessed - SNS by desktop computer and SMA by mobile phone. Users could perceive the greater pervasiveness of mobile phones to decrease stress, because they can get responses no matter where they are - however, users of mobile phones have reported experiencing more stress (Augner \& Hacker, 2012). By beginning to explore the differences in media affordances between an SNS and a SMA, this research hopes to illuminate how the concept of media affordances 
can be used to understand a user's perception of a an SNS versus an SMA, and how that perception affects the user's stress.

This leads to the final set of research questions, which relate to media affordances. The final aim of this study is to determine if any of the measured media affordances affect a user's stress more strongly than the other media affordances. As previously stated, measuring media affordances on an individual basis will allow for results that can be applied broadly across different communication technologies that may exhibit some, but not all, of these media affordances. The measure, adapted from Rice et al. (2017), contains between two and four questions that pertain to different facets of a media affordance, for thirty-two questions in total. Each question asks the user to indicate how his or her perception of the media affordance affects his or her stress - if it is more likely to decrease or increase it. As there are eleven media affordances, each media affordance must be compared to the other ten media affordances to determine if there is a significant difference. If there is a significant difference found between one media affordance and multiple other media affordances, this would indicate that that media affordance affects the user's stress significantly differently than the other affordances. Because each media affordance will be tested for significant difference when compared to each other media affordance, this leads to the following set of research questions:

RQ5a: Given all the media affordances described in the study (eleven in total), which media affordances vary from each other most, in terms of affecting the users' level of stress, for users of the Facebook desktop site? 
RQ5b: Given all the media affordances described in the study (eleven in total), which media affordances vary from each other most, in terms of affecting the users' level of stress, for users of the Facebook mobile application?

RQ6: When asking participants to judge how each media affordance affects their stress, is there a statistically significant difference in how any media affordance affects participants' stress when comparing responses from users of the Facebook desktop site and users of the Facebook mobile application? 


\section{CHAPTER 3: METHODS}

\section{Participants and Recruitment}

After receiving approval to conduct the study from a Human Subjects Review board, this study sought out a nationally representative sample of U.S. adult internet users recruited via Qualtrics, a research platform that coordinates recruitment and data collection for surveys. The data used for this study was part of a larger project examining emotional support processes on four types of media, conducted with five versions of the survey corresponding with four media and one control: the Facebook desktop site, Facebook mobile application, Instagram, Snapchat, and face-to-face communication. To select the sample, Qualtrics randomly selects respondents for the survey where respondents are highly likely to qualify. Qualtrics follows any requests or exclusions requested by the researchers - in the case of this study, the researcher requested a nationally representative sample of U.S. adults, with exclusionary criteria applied in the initial question responses. The exclusionary criteria questions are explained below. Qualtrics proportions each sample from the panel base to the general population, and then randomizes the sample, before deploying the survey. Though there was a best faith effort made to ensure the population was as representative and randomized as possible, there is an element of convenience due to the versions of the survey closing once the version reached a threshold of 200 respondents, as well as self-selection due to the sorting question.

Qualtrics was compensated to recruit and collect this study's data. Qualtrics, in turn, compensated participants who completed the survey with credit that varies based on 
their panelist profile and target acquisition difficulty. The specific type of reward varies, and may include cash, airline miles, gift cards, redeemable points, sweepstakes entries, and vouchers. All participants who completed the survey were compensated equally according to the Qualtrics reward system.

The research team set up all five versions of the survey within Qualtrics, and instructed Qualtrics to collect 200 responses per survey, at which point it would automatically stop collection (there was an accidental oversampling of the Facebook mobile survey, which resulted in 297 total participants). In order to determine which version of the survey participants would take, and ensure that they answered questions about the platform they were most familiar with, they were asked a sorting question that asked, "Of the following options, how do you most frequently reach out to others when you feel stressed?" with five options corresponding to the five versions of the survey: $1=$ Facebook desktop site, 2 = Facebook mobile application, $3=$ Instagram, $4=$ Snapchat, 5 = Face to face conversations. Depending on the option selected, the participant was then directed to the corresponding version of the survey. This study examined only the data from the Facebook desktop site and Facebook mobile application versions of the study. Participants who successfully completed the survey were thanked and instructed how to obtain compensation.

Participants were excluded from the survey if they were not 18 years old or older. They were also excluded if they declined to accept the terms of the informed consent, which described the purpose of the study as "how using social media or interacting faceto-face with another person or other people affects a person's stress" (see Appendix A for 
the informed consent form). It also explicitly stated that participation was voluntary and anonymous. Finally, there was a measure of how often participants used communication channels for support when stressed, asking, "When you are feeling stressed, how often do you reach out to others in the following ways?" There were 16 total options, including face-to-face one-to-one conversations, Public Facebook desktop site post, and private message via Instagram, with answer choices ranging from $1=$ Never to $9=$ many times $a$ day (full measure discussed in the measures section below). If participants responded that they never reached out by face-to-face communication, they were excluded from the survey. This was due to an aim of the larger study, which requires participants to reach out by face-to-face communication.

Demographics were collected as part of the larger project, and while demographics were not included in the research questions of this study, it is relevant that the demographics of the two population samples are similar. Randomization of data collection is vital for allowing comparisons between two populations, but having similar demographics also helps strengthen the argument for comparing two populations. Aside from a stronger majority of female participants in the Facebook mobile application sample, the populations are fairly similar. The Facebook desktop site sample consisted of 201 total participants, $44.8 \%$ male $(n=90)$ and $55.2 \%$ female $(n=111)$. The participants' mean age range was $51.0(S D=15.65)$, with a range of 21 to 80 . Ethnicity of respondents included 79.1\% White/Anglo/Caucasian/Middle Eastern $(\mathrm{n}=159), 9.0 \%$ Black/African American $(n=18), 4.5 \%$ Asian $(n=9), 0.1 \%$ American Indian or Alaskan Native $(n=2)$, 4.5\% Hispanic or Latino $(n=9)$, and $2.0 \%$ Other $(n=4)$. Overall, most participants 
within this sample work full time $(35.1 \%, n=71)$ or are unemployed or retired $(27.2 \%, n$ $=55)$. The remaining participants work part time $(13.4 \%, n=27)$, are home makers $(11.4 \%, n=23)$, are unable to work $(10.9 \%, n=22)$, or are undergraduate students $(0.9 \%, n=2)$ or graduate students $(0.49 \%, n=1)$.

The Facebook mobile application sample consisted of 297 total participants, $29.6 \%$ male $(n=88), 70.0 \%$ female $(n=208)$, and $0.3 \%$ other $(n=1)$. The participants' mean age range was $41.6(S D=13.72)$, with a range of 19 to 79 . Ethnicity of respondents included 80.0\% White/Anglo/Caucasian/Middle Eastern $(\mathrm{n}=235), 8.1 \%$ Black/African American $(\mathrm{n}=24), 5.4 \%$ Asian $(\mathrm{n}=16), 1.0 \%$ American Indian or Alaskan Native $(\mathrm{n}=$ 3), 5.4\% Hispanic or Latino $(n=16)$, and $0.3 \%$ Other $(n=1)$. Overall, most participants within this sample work full time $(35.1 \%, n=126)$. The remaining participants work part time $(13.4 \%, n=27)$, are home makers $(11.4 \%, n=23)$, are unemployed or retired, $(11.4 \%, n=11)$, are unable to work $(10.9 \%, n=22)$, or are undergraduate students $(0.9 \%, n=2)$ or graduate students $(0.49 \%, n=1)$.

\section{Procedure}

As noted above, there was first a sorting question and a measure asking how often, and in what ways, participants reported reaching out to others when stressed. In order to determine which version of the survey participants would take, and ensure that they answered questions about the platform they were most familiar with, the sorting question asked, "Of the following options, how do you most frequently reach out to others when you feel stressed?" with five options corresponding to the five versions of the survey: 1 = Facebook desktop site, 2 = Facebook Mobile application, 3 = Instagram, 
$4=$ Snapchat, $5=$ Face to face conversations. The option selected would result in the participant being directed to the corresponding version of the survey. Participants were also asked about their use of communication channels to reach out for support when stressed. This was adapted from a measure used by Rice and colleagues (2017). They indicated this with a Likert-type scale ranging from $1=$ Never to $9=$ Many times a day. There were 18 questions in this measure, and the options were separated by media and by type of action, including "Public Facebook post via the mobile application," "Private message via Instagram," and "Face to face, one-on-one conversations." If the participant indicated that they never reached out for face-to-face emotional support, they were excluded from the survey. This was implemented because of a research goal of the larger overall study.

A brief trial run was conducted on Friday, March 30 to confirm that the sorting mechanism was working properly and there were no discrepancies. After determining that this was the case, data collection resumed on Thursday, April 5, and ended Friday, April 27, when each version of the survey reached 200 participants. At this point, data cleaning was conducted to separate out any participants that demonstrated straight lining or patterned behavior, or provided "gibberish" responses to the open-ended questions. Straight lining behavior was defined as choosing the same number for at least $90 \%$ of a measure. Patterned behavior was defined as choosing alternating numbers, or numbers in a clear sequence (i.e. 1, 2, 3, 4, 5) for at least $90 \%$ of a measure. Gibberish responses were defined as any response in the open-ended text box that did not make sense within the context of the question. Participants had to demonstrate straight lining or patterned 
behavior for at least three of the four measures to be considered for exclusion. The final list of participants was given to Qualtrics, who reviewed the list of provided responses and agreed to replace them. Qualtrics was contracted to sample each version until the total reached 200, however, an accidental oversampling occurred of the Facebook mobile version. With the removal of the agreed-upon respondents, the total respondents for the Facebook mobile version still exceeded 200, so no respondents were replaced. Within the Facebook desktop version, Qualtrics agreed to replace 44 participants. Resampling began on May 2 and ended on May 11.

\section{Measures}

All measures were included in both the Facebook desktop site and Facebook mobile application versions of the survey. The only difference between the two versions was any reference to the relevant survey (i.e. the Facebook desktop site survey referred to the Facebook desktop site within the questions, and the Facebook mobile application survey referred to the Facebook mobile application within the questions). The measures below will all use the Facebook desktop site within the wording. The measures below also appear in the order the participants saw them.

Communication Channels. This was the first measure within the survey. This measure was adapted from Rice et al. (2017) and used to determine which communication channels participants utilized for support when feeling stressed. As discussed in the literature review, this measure captures the frequency that participants are utilizing media, but instead of overall use, it attends to a specific action: reaching out for support when feeling stressed. It includes 16 channels: 1) face-to-face one-to-one 
conversations, 2) face to face conversations with more than one person, 3)

sending/receiving emails, 4) telephone (landline or mobile) calls, 5) short messages (including text messages, google chat, other chat programs), 6) video calls (Google hangouts, Facetime, other video communication), 7) Public Facebook computer site post, 8) Private Message via Facebook computer site, 9) Public Facebook mobile app post, 10) Private Message via Facebook mobile app, 11) Public Instagram post, 12) Private message via Instagram, 13) Public Reddit post, 14) Private message via Reddit, 15) Public Snapchat post, and 16) Private message via Snapchat. Participants were asked to indicate how often they used each channel using a 9-point Likert-type scale as follows: 1 $=$ Never, $2=$ A few times a year or less, $3=$ Once a month or less, $4=$ A few times $a$ month, $5=$ Once a week, $6=$ A few times a week, $7=$ Every day, $8=$ A few times a day, 9 = Many times a day.

Reliability was conducted for this scale for each population. The resulting Cronbach's Alpha for the Facebook desktop site population was $\alpha=.965$, and for the Facebook mobile application population was $\alpha=.961$. This measure can also be found in Appendix B.

Perceived Stress. Participants were asked about their perceived life stress using the 10item Perceived Stress Scale (PSS) developed Cohen, Kamarck, and Mermelstein (1983). Instead of measuring stress as a specific point, this measure deals with a more general measure of overall perceived stress. The measure is meant to determine the degree to which participants found situations in their life to be stressful over the previous month. Following is the list of 10 questions: 
1. In the last month, how often have you been upset because of something that happened unexpectedly?

2. In the last month, how often have you felt that you were unable to control the important things in your life?

3. In the last month, how often have you felt nervous and "stressed"?

4. In the last month, how often have you felt confident about your ability to handle your personal problems? (reverse)

5. In the last month, how often have you felt that things were going your way? (reverse)

6. In the last month, how often have you found that you could not cope with all the things you had to do?

7. In the last month, how often have you been able to control irritations in your life? (reverse)

8. In the last month, how often have you felt that you were on top of things? (reverse)

9. In the last month, how often have you been angered because of things that were outside of your control?

10. In the last month, how often have you felt difficulties were piling up so high that you could not overcome them?

Each question was measured on a 5-part Likert-type scale as follows: $0=$ Never, 1 $=$ Almost Never, $2=$ Sometimes, $3=$ Fairly Often, $4=$ Very Often, , with questions that have "(reverse)" reverse coded. Reliability was conducted for this scale for each population. The resulting Cronbach's Alpha for the Facebook desktop site population was $\alpha=.813$, and for the Facebook mobile application population was $\alpha=.785$. This measure can also be found in Appendix C.

Media Affordances. This measure was adapted from Rice et al.'s (2017) measure of organizational media affordances, and the lead researcher for the larger project worked with Ronald Rice to adapt the measure to how the media affordances affect stress. This measure asks about eleven affordances: association, awareness, editability, persistence, personalization, pervasiveness, searchability, sharing, evaluability, visibility, and signaling. The measure asks questions that pertain to facets of each affordance, with 
approximately two to four questions per affordance. The measure contains the following

32 questions:

The following questions were asked relating to the affordance "association:"

1. When I find information I already knew or was aware of while using the Facebook desktop site, it is more likely to...

2. When I find new information I did not know or wasn't aware of while using the Facebook desktop site, it is more likely to...

3. When I find people I already know or am aware of while using the Facebook desktop site, it is more likely to...

4. When I find new people I did not know or wasn't aware of while using the Facebook desktop site, it is more likely to...

The following questions were asked relating to the affordance "awareness:"

5. When I become aware of the information others have while using the Facebook desktop site, it is more likely to...

6. When I become aware of activities, opinions, or locations of others while using the Facebook desktop site, it is more likely to...

7. When I keep up-to-date with what others are posting on the Facebook desktop site, it is more likely to...

8. When I keep up-to-date with the Facebook desktop site policies and norms, it is more likely to...

The following questions were asked relating to the affordance "editability:"

9. When I edit others' content (i.e. deleting comments) after they have posted it on the Facebook desktop site, it is more likely to...

10. When I edit my content (i.e. editing or deleting posts, comments, etc.) on the Facebook desktop site after I have posted it, it is more likely to...

11. When I create or edit a post along with other people on the Facebook desktop site, it is more likely to...

The following questions were asked relating to the affordance "persistence:"

12. When I maintain relations with others on the Facebook desktop site despite changes in activities or location, it is more likely to...

13. When I have my information or comments stay available after I post them on the Facebook desktop site, it is more likely to...

The following questions were asked relating to the affordance "personalization:" 
14. When I include information that presents my personal identity on the Facebook desktop site, it is more likely to...

15. When I include photos that present my personal identity on the Facebook desktop site, it is more likely to...

16. When I include other content that presents my personal identity on the Facebook desktop site, it is more likely to...

The following questions were asked relating to the affordance "pervasiveness:"

17. When I get responses to my requests from others quickly while using the Facebook desktop site, it is more likely to...

18. When I communicate with others on the Facebook desktop site while moving, commuting, or traveling, it is more likely to...

The following questions were asked relating to the affordance "searchability:"

19. When I search for information or people by entering search words on the Facebook desktop site, it is more likely to...

20. When I search for information or people by following links between content on the Facebook desktop site, it is more likely to...

The following questions were asked relating to the affordance "sharing:"

21. When I search for tags or keywords that someone else has added to content on the Facebook desktop site, it is more likely to...

22. When I create groups for sharing information about thoughts, feelings, concerns, ideas, etc. on the Facebook desktop site, it is more likely to...

23. When I share my posts, updates, photos, videos, and other types of content with other people on the Facebook desktop site, it is more likely to ...

The following questions were asked relating to the affordance "evaluability:"

24. When I see other people's evaluation of information through their recommendations on the Facebook desktop site, it is more likely to...

25. When I see other people's evaluation of information through their comments on the Facebook desktop site, it is more likely to...

26. When I see other people's evaluation of information through their liking on the Facebook desktop site, it is more likely to...

27. When I see other people's evaluation of information through their tagging on the Facebook desktop site, it is more likely to...

The following questions were asked relating to the affordance "visibility:"

28. When I see other people's answers to other people's questions on the Facebook desktop site, it is more likely to... 
29. When I see interactions or links with other people on the Facebook desktop site, it is more likely to...

30. When I see the number of others who have "liked" or linked to the same content on the Facebook desktop site, it is more likely to...

The following questions were asked relating to the affordance "signaling:"

31. When I receive notifications about other information or updates that are similar to what I have just been looking at on the Facebook desktop site, it is more likely to...

32. When I receive notifications about other people's information or updates on the Facebook desktop site, it is more likely to....

To anchor these media affordances in how they affect perceptions of stress, each question asked how a scenario affected the participant's stress within a 7-point Likerttype scale: 1 = Strongly increase my stress, 2 = Increase my stress, 3 = increase my stress a little bit, 4 = not increase or decrease my stress, $5=$ decrease my stress a little bit, $6=$ decrease my stress, 7 = strongly decrease my stress. Two additional options were included, and participants were instructed to choose " $8=$ Not possible" if they did not believe the scenario was possible on the platform, and "9= Do not know" if they did not know how the scenario affected their stress; these were treated as missing. Reliability was conducted for this scale for each population. The resulting Cronbach's Alpha for the Facebook desktop site population was $\alpha=.943$, and for the Facebook mobile application population was $\alpha=.955$. This measure can also be found in Appendix D.

Perceived Emotional Support. In 1996, Weber and Patterson developed and tested a Communication Based Emotional Support Scale (CBESS) to measure perceptions of communication-based emotional support from romantic partners, and the researchers define their scale as being directly concerned with support as a product of interpersonal relationships. Although it was originally intended to be used to measure 
perceived emotional support from romantic partners, it has been successfully applied to many different contexts, including SNSs.

Participants were asked to what extent they agree with 20 statements that address different facets of perceived emotional support, using a 5-point Likert-type scale: $1=$ Almost Never True, $2=$ Rarely True, $3=$ Occasionally True, $4=$ Often True, $5=$ Almost Always True. The questions were adapted to determine if participants perceive emotional support to be available either on the Facebook desktop site or the Facebook and Facebook Messenger applications. The questions are as follows, with questions that have “(reverse)" reverse coded:

1. There are people on the Facebook desktop site that help me work through my thoughts and feelings about major life decisions (e.g. career choice)

2. There are people on the Facebook desktop site that will do things that they know will upset me (reverse)

3. There are people on the Facebook desktop site that patiently and sensitively listen to me "let off steam" about an outside problem that I am having

4. When I tell people on the Facebook desktop site about a problem that I am having, they don't seem to be paying attention (reverse)

5. There are people on the Facebook desktop site that help me cope with problems concerning other friends and/or family members

6. People on the Facebook desktop site avoid me when I am depressed (reverse)

7. There are people on the Facebook desktop site messenger that are good listeners when I am upset

8. When I tell people on the Facebook desktop site about a problem that I am having, they respond with "If you think that is bad, listen to this..." (reverse)

9. There are people on the Facebook desktop site that never listen to my problems (reverse)

10. There are people on the Facebook desktop site that say and do supportive things for me when I am feeling down

11. When I want to talk to people on the Facebook desktop site about what is bothering me, they seem to have something else to do (reverse)

12. There are people on the Facebook desktop site that show genuine concern for my problems

13. When I talk to people on the Facebook desktop site about a problem that I have, they tell me that I am overreacting (reverse) 
14. There are people on the Facebook desktop site that give me good advice when I ask for it

15. I don't like to talk about things that are bothering me with people on the Facebook desktop site because they will think that I am mad at them and get defensive (reverse)

16. There are people on the Facebook desktop site that make it very easy to discuss my personal feelings

17. There are people on the Facebook desktop site that tell me what I should do even when I don't ask for advice (reverse)

18. There are people on the Facebook desktop site that listen to my side of the story even if they think that I am wrong

19. There are people on the Facebook desktop site that don't understand that when I am in a bad mood, sometimes I just need to "blow off steam" (reverse)

20. There are people on the Facebook desktop site that make an effort to make me feel better when I am depressed

Reliability was conducted for this scale for each population. The resulting Cronbach's Alpha for the Facebook desktop site population was $\alpha=.806$, and for the Facebook mobile application population was $\alpha=.794$. This measure can also be found in Appendix E.

Demographics. Questions were asked to determine the demographics of participants. The first question asked "What is your current job status? Please pick the category that best describes your current job status.” Answer choices were: Working full time, Working part time, Graduate student, Undergraduate student, Home maker, Unable to work, and Unemployed/Retired. The second question asked, "What is your ethnicity?" with answer options as follows: White/Anglo/Caucasian/Middle Eastern, Black/African American, Asian, American Indian or Alaskan Native, Hispanic or of Latino origin, and Other, please specify with an open text box. The third question asked what year participants were born, with a drop-down menu that offered years ranging from 1900 to 2001. Finally, participants were asked "What is your sex?" with answer options: Male, Female, and Other with an open text box. This measure can also be found in Appendix F. 


\section{CHAPTER 4: RESULTS}

Research Question 1 asked when looking at participants' personal judgment of life stress, if users of the Facebook desktop site or users of the Facebook mobile application were more likely to report greater stress? To determine this, a mean score was calculated from the Perceived Stress Scale, and an independent samples t-test compared mean scores between the Facebook desktop site sample and Facebook mobile application sample. It was found that those who chose to answer questions about the Facebook mobile application $(M=3.05, S D=.59)$ reported slightly higher general life stress than those who chose to answer questions about the Facebook desktop site $(M=2.91, S D=$ .69) $[t(384)=-2.36, p=0.019, r=.11]$. A Levene's test indicated unequal variances $(\mathrm{F}=$ $6.35, p=.012$ ), so degrees of freedom were adjusted from 497 to 384 .

Research Question 2 asked: when looking at participants’ personal judgment of perceived emotional support on the platform they indicated they used most to reach out for emotional support, which group was more likely to report higher perceptions of emotional support on that platform: users of the Facebook desktop site, or users of the Facebook mobile application? To determine this, a mean score was calculated from the Communication Based Emotional Support Scale, and an independent samples t-test compared means between each sample. No significant differences were found between the Facebook mobile application sample $(M=3.29, S D=.48)$ and the Facebook desktop site sample $(M=3.35, S D=.53)[t(497)=1.24, p=.215]$.

Research Question 3a asked if there was a correlation between general life stress and perceived emotional support available on the Facebook desktop site. There was no 
significant correlation between general life stress and perceived emotional support for the Facebook desktop site sample, Pearson's $r=-.11, p=.117$. In other words, as general life stress increased, perceived emotional support did not increase or decrease.

Research Question $3 b$ asked if there was a correlation between perceived stress and perceived emotional support available on the Facebook mobile application. There was also no significant correlation between general life stress and perceived emotional support for the Facebook mobile application sample, Pearson's $r=-.072, p=.213$. In summary, regardless if participants perceived the Facebook desktop site or mobile application to have high levels of emotional support available, it did not increase or decrease their perceived life stress.

Research Question 4a asked if there was a correlation between perceived emotional support and frequency of using the Facebook desktop site to reach out for emotional support. Perceived emotional support was determined by calculating a mean score from the Communication Based Emotional Support Scale, in which a higher score indicates a higher perception of emotional support. Frequency of reaching out for emotional support was measured two ways, by private messaging and public posting on each platform. A higher score indicates reaching out more frequently. First, there was a negative correlation between perceived emotional support and reaching out by public post on the Facebook desktop site. In other words, participants within the Facebook desktop site sample reached out by public post significantly less frequently if they perceived a higher level of emotional support to be available to them, Pearson's $r=-.221$, $p=.002$. Second, there was also a negative correlation between perceived emotional 
support and reaching out by private message on the Facebook desktop site. Participants within the Facebook desktop site sample reached out by private message significantly less frequently if they perceived a higher level of emotional support to be available to them, Pearson's $r=-.168, p=.017$.

Research Question 4b asked if there was a correlation between perceived emotional support and frequency of using the Facebook mobile application to reach out for emotional support, with perceived emotional support and frequency calculated in the same way as in RQ4a. For the Facebook mobile application sample, there was a negative correlation between perceived emotional support and reaching out by public post. Participants reached out significantly less frequently by public post if they perceived a higher level of emotional support to be available to them on the platform, Pearson's $r=-$ $.156, p=.007$. However, there was no correlation between frequency of reaching out by private message and perception of emotional support on the platform, Pearson's $r=-.024$, $p=.686$.

Research Question 5a asked: Given all the media affordances described in the study (eleven in total), which media affordances to participants say are more likely to affect their stress when comparing the media affordances to each other, for users of the Facebook desktop site? Research Question $5 b$ asked the same question, but for users of the Facebook mobile application. As discussed, the media affordance measure uses Rice et al.'s (2017) aggregation of 11 organizational media affordances, with questions adapted from the original to be applied to social media and stress. Within the adapted media affordance measure, questions are asked that pertain to each media affordance, 
with two to four questions per media affordance, making a total of 32 questions for the measure.

The following was done for each sample: questions that pertained to each media affordance were combined to create a mean score for that media affordance. For example, questions 1-4 in the media affordance measure pertained to the media affordance "association," and were combined to create a mean score for the media affordance “association." With 11 media affordances, that created 11 mean scores. Then, each media affordance mean score was compared to the 10 other media affordance mean scores, using paired t-tests. This resulted in a total of 55 paired t-tests for each sample. The full table of results can be found in Table 2 and 3 below. However, following the reported results for research questions $5 \mathrm{a}$ and $5 \mathrm{~b}$, additional tables will be provided that show only the relevant results from this larger table, for clarity. 


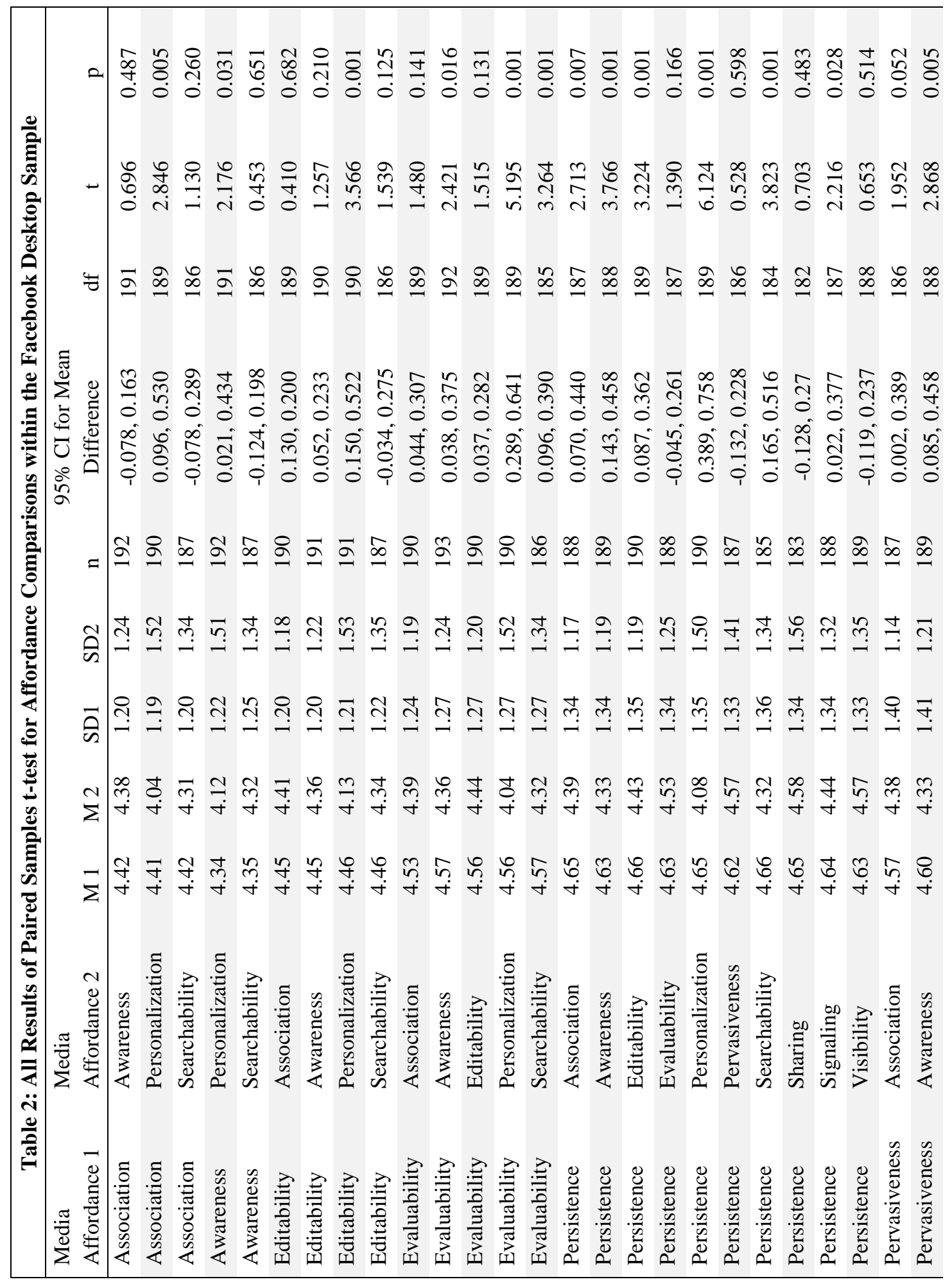




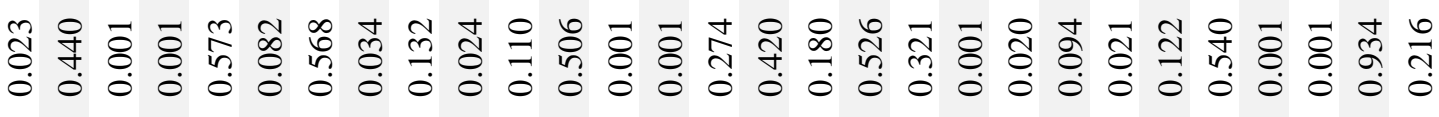

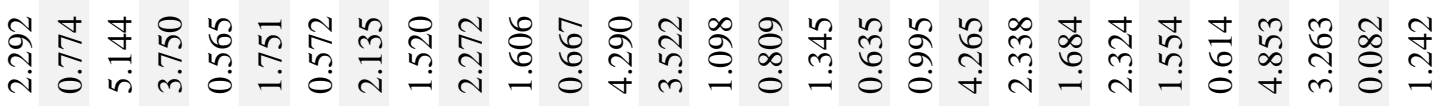

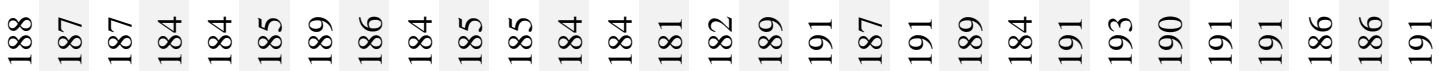

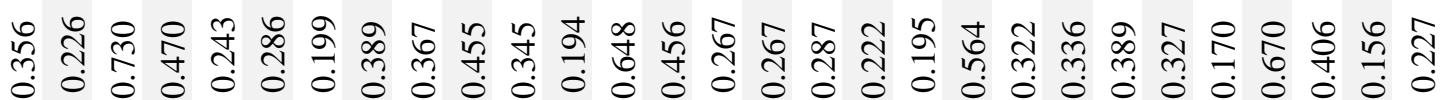

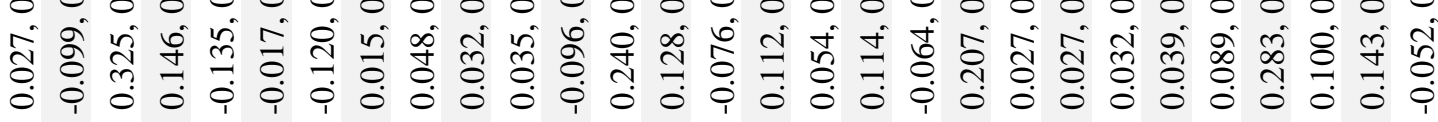

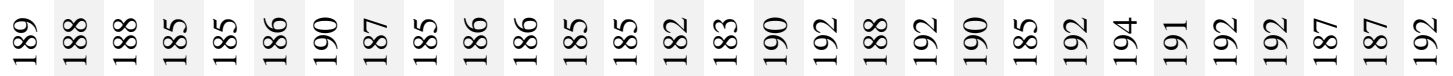

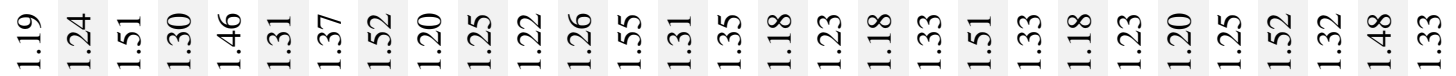

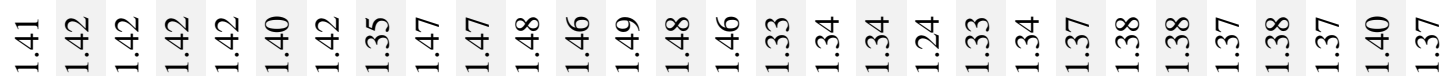

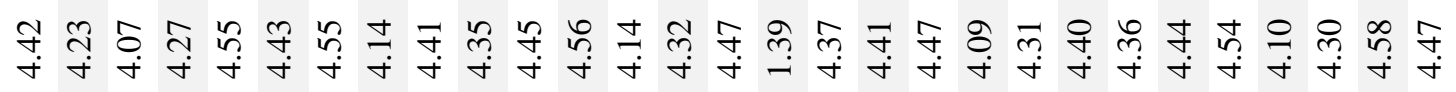

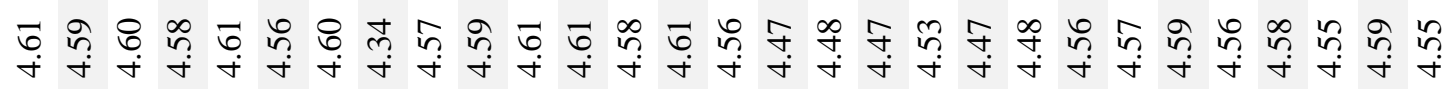
MIIIIIIIIIIUIIII

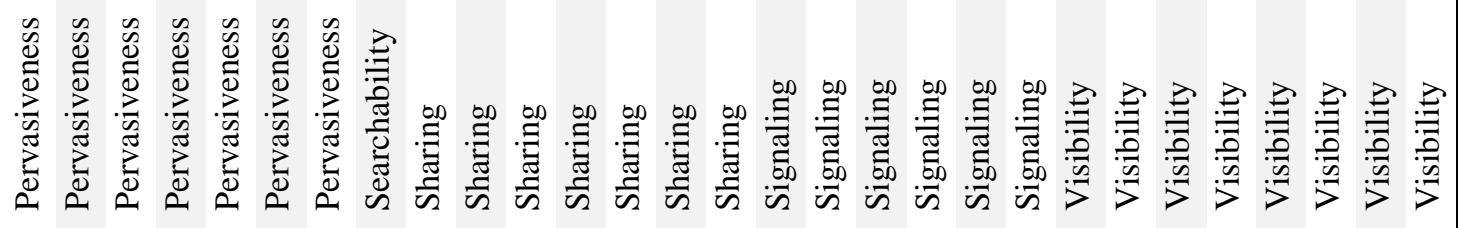




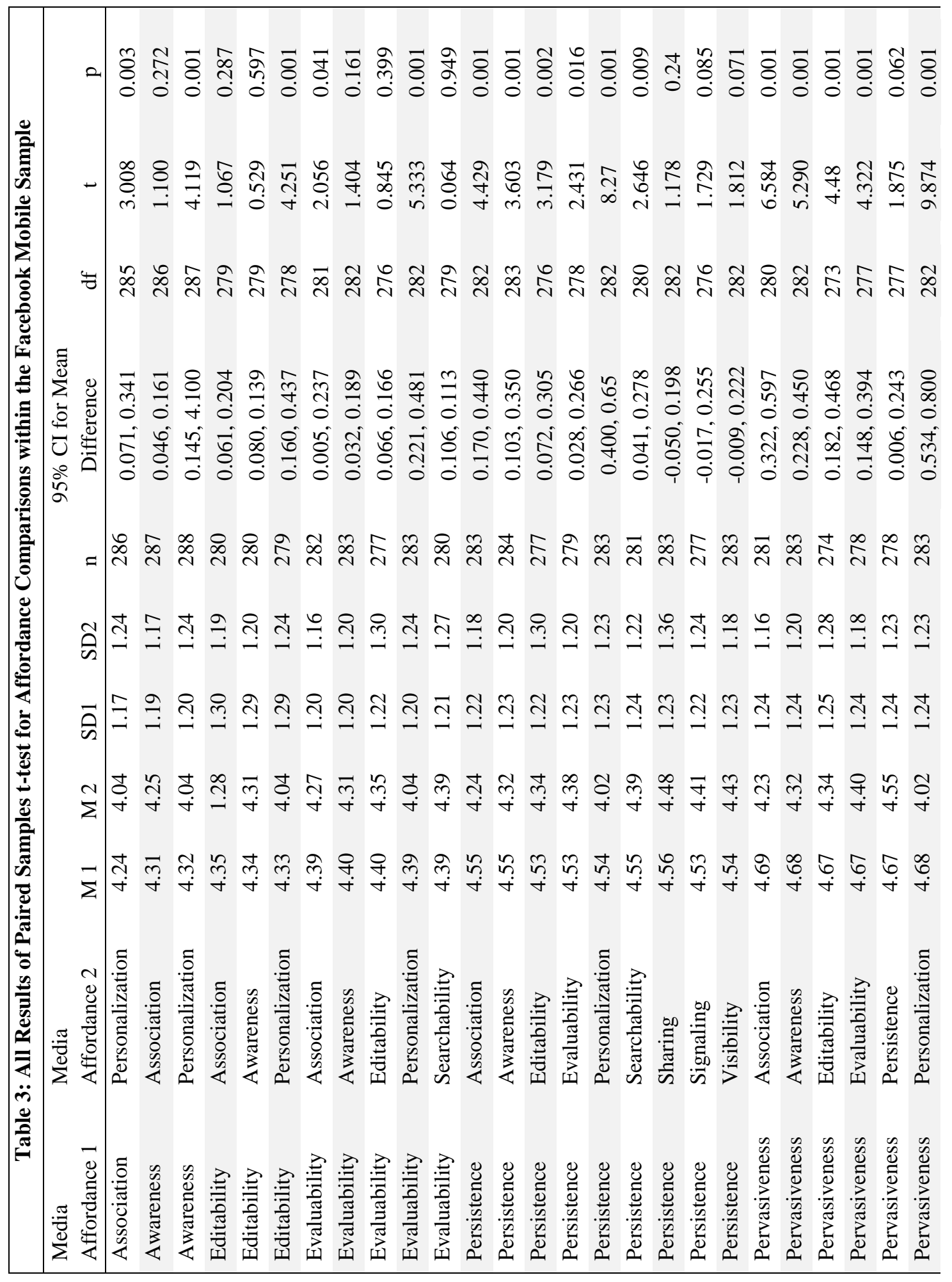




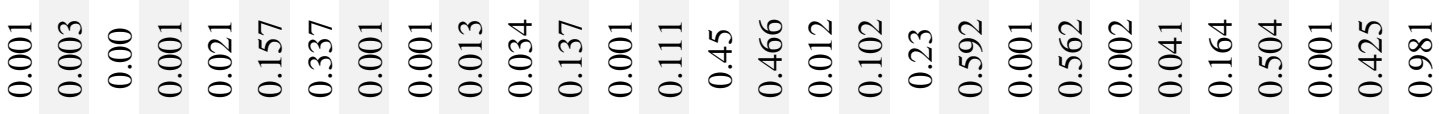

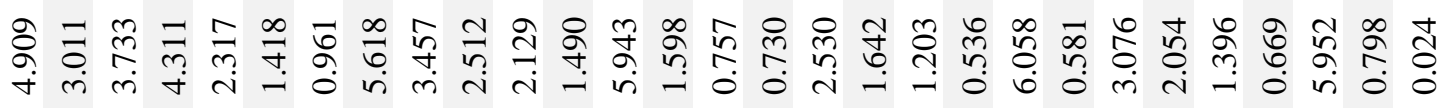

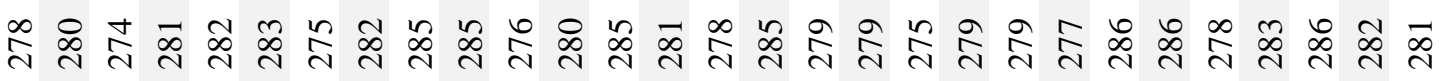

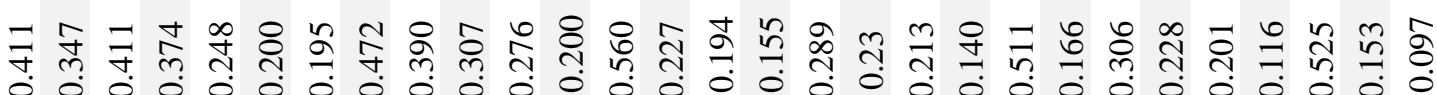

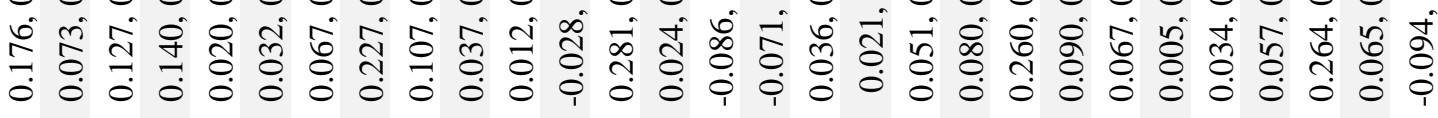

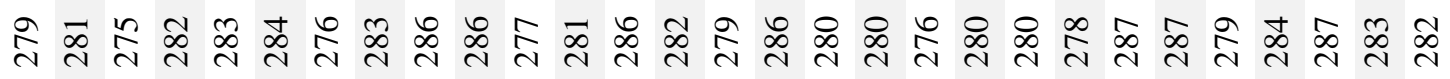

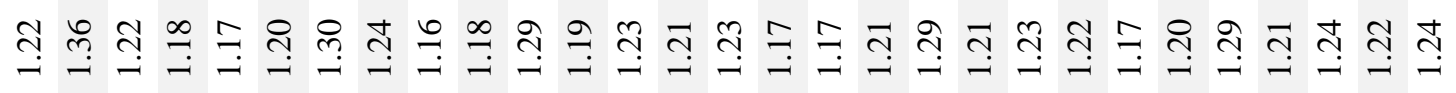

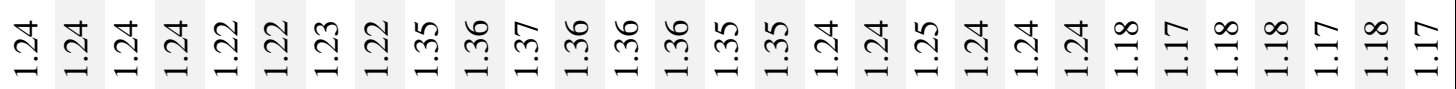

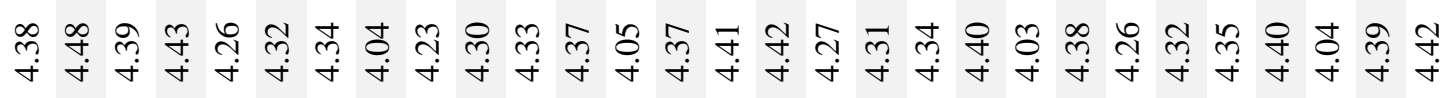

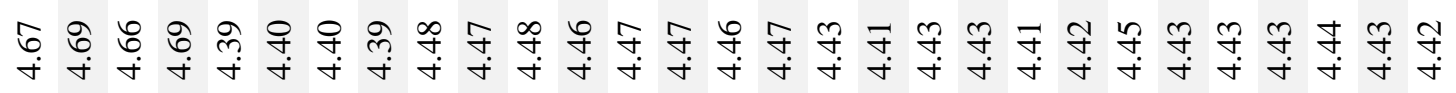

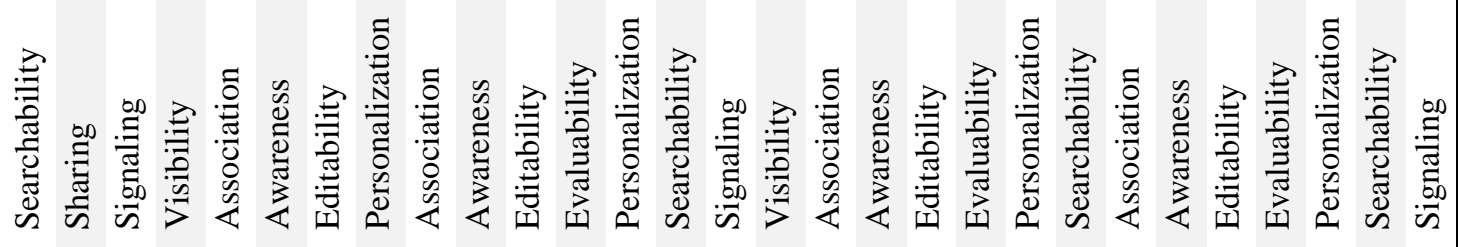

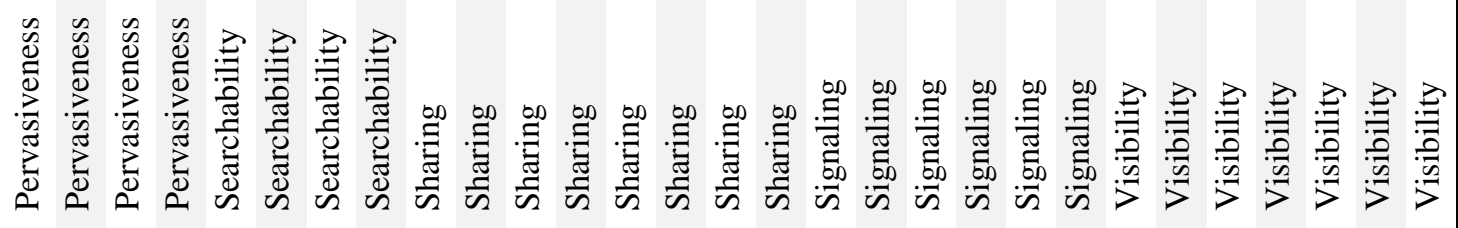


To summarize, RQ5a and 5b were interested in whether there were differences, on balance, when looking at and comparing the mean scores of each media affordance. With 11 total media affordances, after each media affordance was compared to the other 10 , the responses needed to be sorted in a way that made the results make sense contextually. If one media affordance had a significantly higher or lower mean than many of the other media affordances, indicating that it was more likely to be associated with feelings of decreased stress or increased stress, that could indicate that the perception of that media affordance stands out in participants' minds as having a stronger effect on their stress than the other media affordances. To report the results in a way that is holistic and captures the spirit of the research questions, the results were organized by which media affordances stood out as having a statistically higher or lower mean score than many of the other media affordances

For RQ5a, which was within the Facebook desktop sample, persistence and pervasiveness both had means significantly higher than many of the other media affordances they were compared to. The scale for the measure ranges from $1=$ Strongly increase my stress to $7=$ strongly decrease my stress, therefore, a higher mean indicates a likelihood to decrease stress. Persistence had a significantly higher mean than six of the other media affordances: association, awareness, editability, personalization, searchability, and signaling. Pervasiveness had a significantly higher mean than four of the other media affordances: awareness, editability, personalization, and searchability; note that this is not a majority, but is contextually relevant for reasons that will be explained in the discussion section. These findings can be found in Table 4a. 
In contrast, a media affordance with a significantly lower mean would indicate a likelihood to increase stress. Personalization was found to have a significantly lower mean than all ten other media affordances: association, awareness, editability, evaluability, persistence, pervasiveness, searchability, sharing, signaling, and visibility. Searchability had a significantly lower mean than six of the other ten media affordances: evaluability, persistence, pervasiveness, sharing, signaling, and visibility. These findings can be found in Table $4 b$. 


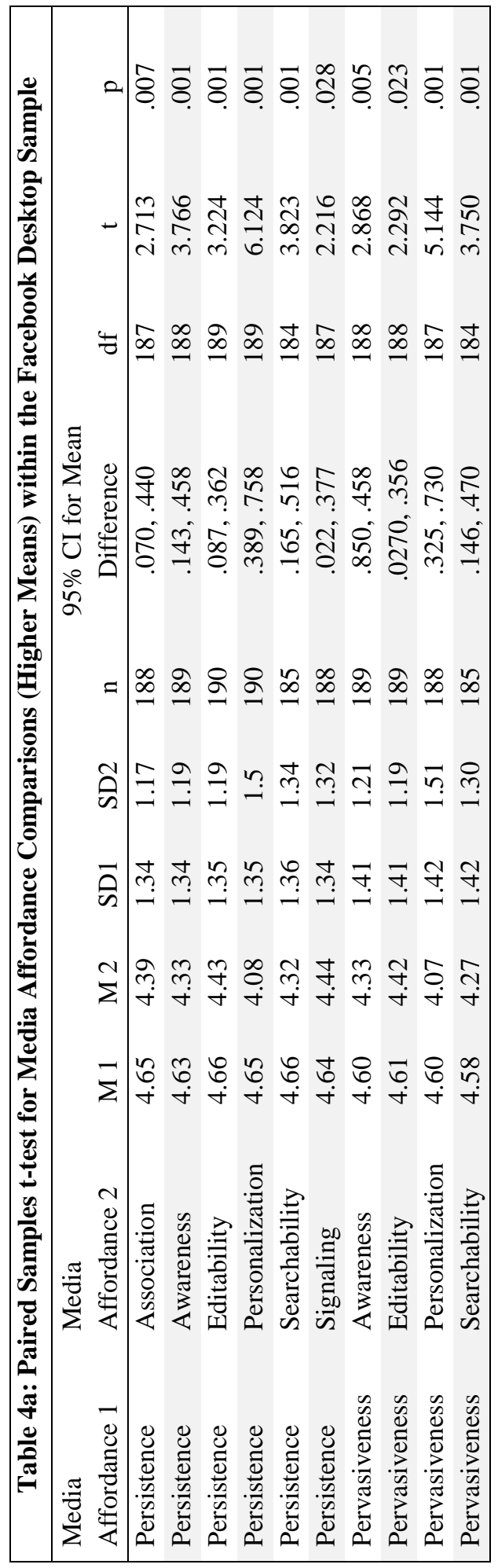




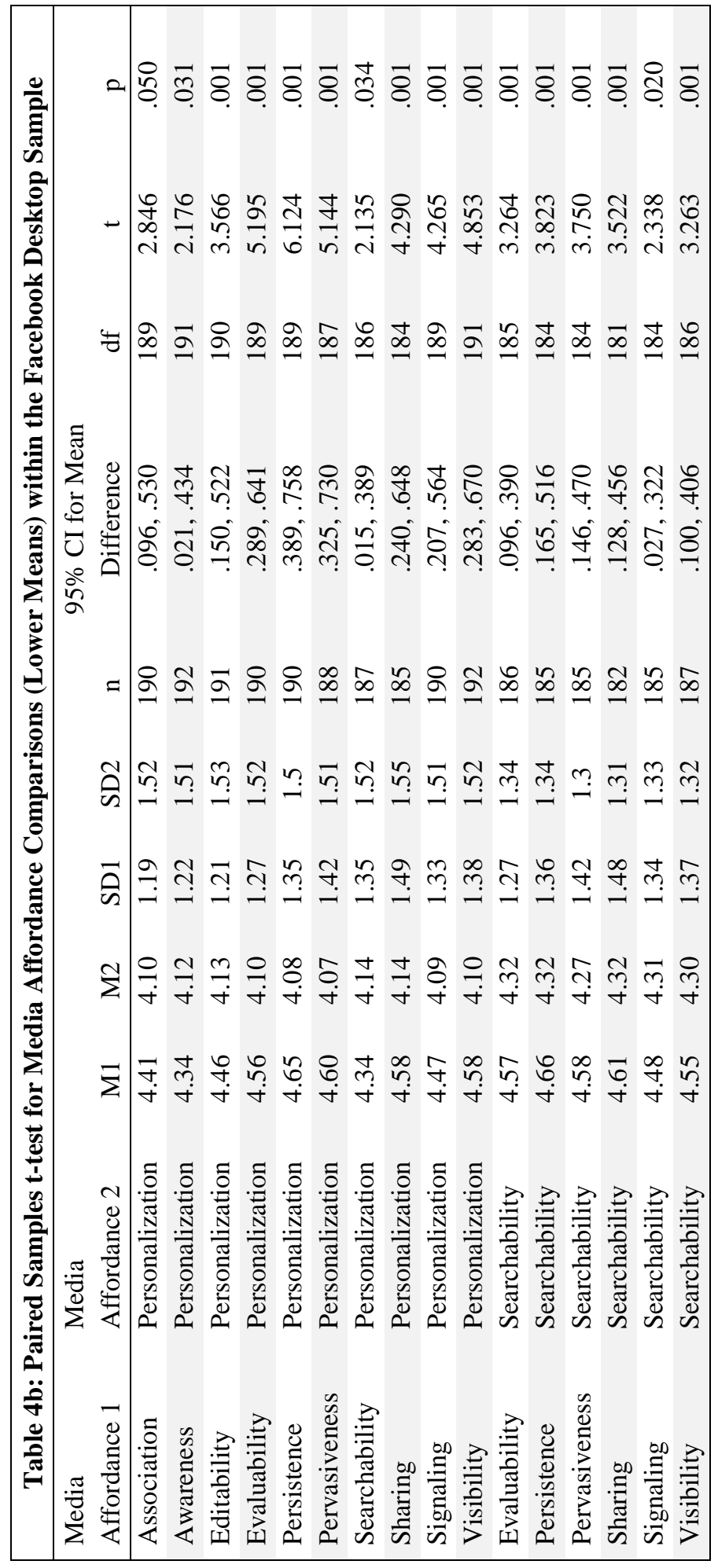


For RQ5b, which was within the Facebook mobile application sample, pervasiveness and persistence both had means significantly higher than many of the other media affordances they were compared to. Pervasiveness had a mean significantly higher than nine of the ten other media affordances: association, awareness, editability, personalization, searchability, sharing, evaluability, visibility, and signaling (all except persistence). Persistence had a significantly higher mean than six of the ten other media affordances: association, awareness, editability, personalization, searchability, and evaluability. These findings are in Table 5a.

Still within the Facebook mobile sample, there were two media affordances with significantly lower means than many of the other media affordances. Personalization had a significantly lower mean than all ten other media affordances: association, awareness, editability, evaluability, persistence, pervasiveness, searchability, sharing, signaling, and visibility. Association had a significantly lower mean than seven of the ten other media affordances: evaluability, persistence, pervasiveness, searchability, sharing, signaling, and visibility. These findings are in Table $5 \mathrm{~b}$. 


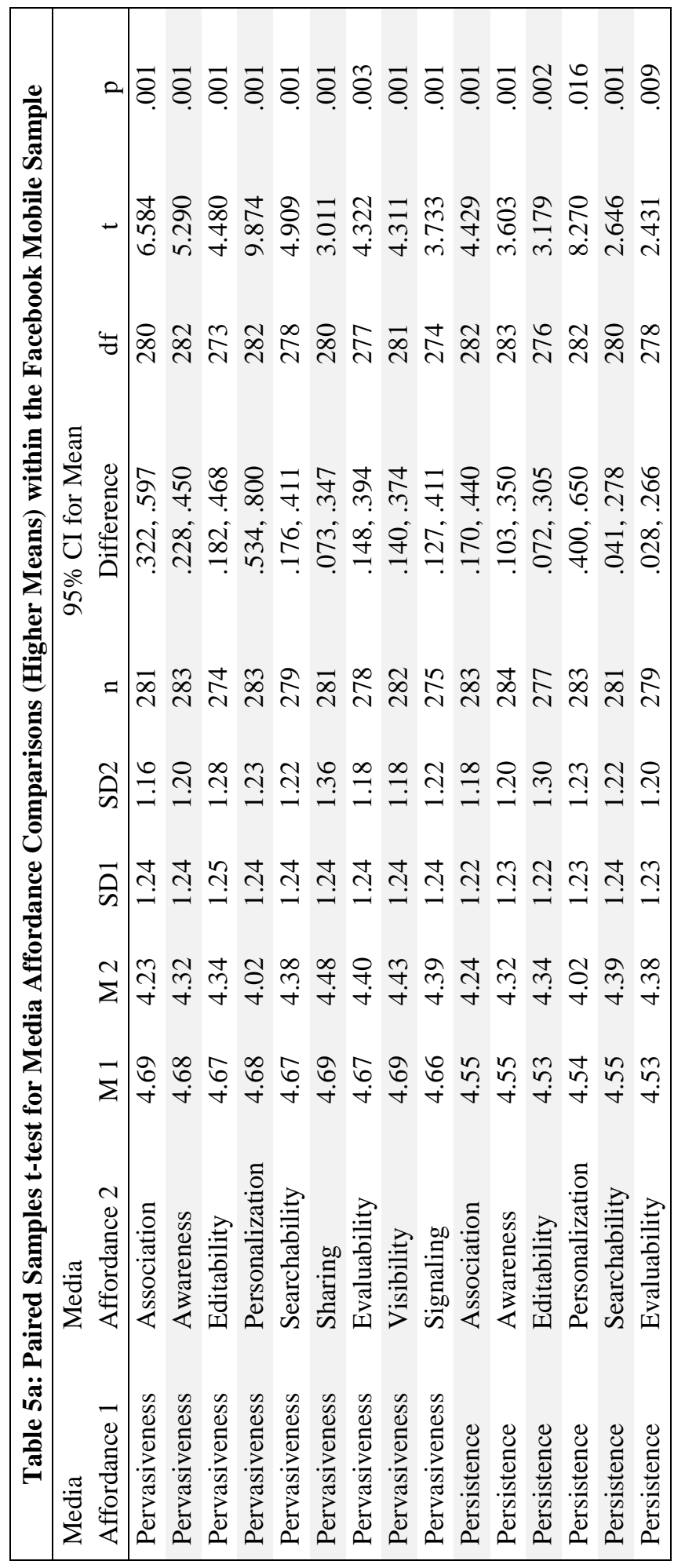




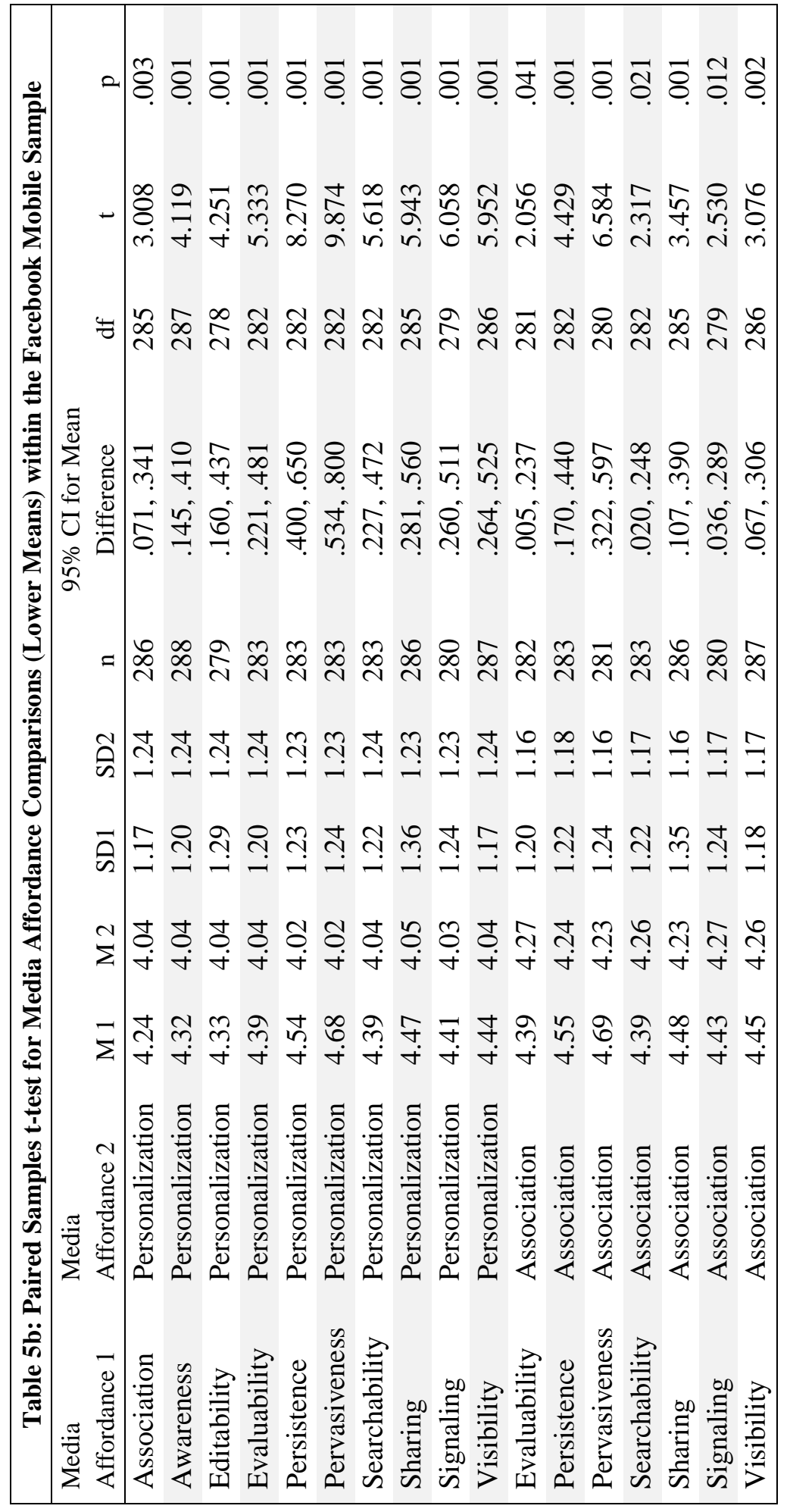


Research Question 6 also addressed the media affordance measure - it asked, when asking participants to judge how each media affordance affected their stress, if there was a significant difference in how any media affordance affected stress when comparing responses from users of the Facebook desktop site and Facebook mobile application. For example, after arriving at a mean score for the media affordance "association" within the Facebook desktop site sample, is there a significant difference from the mean score for "association" within the Facebook mobile application sample? To determine this, the questions that pertained to each media affordance were combined to create a mean score for that media affordance. For example, questions 1-4 in the media affordance measure pertained to the media affordance "association," and were combined to create a mean score for the media affordance "association." With 11 media affordances, that created 11 mean scores. Then, an independent samples t-test compared each media affordance between the Facebook desktop site sample and the Facebook mobile application sample. There was no significant difference found in the mean scores of any of the media affordances. This data can be found in Table 6 . 


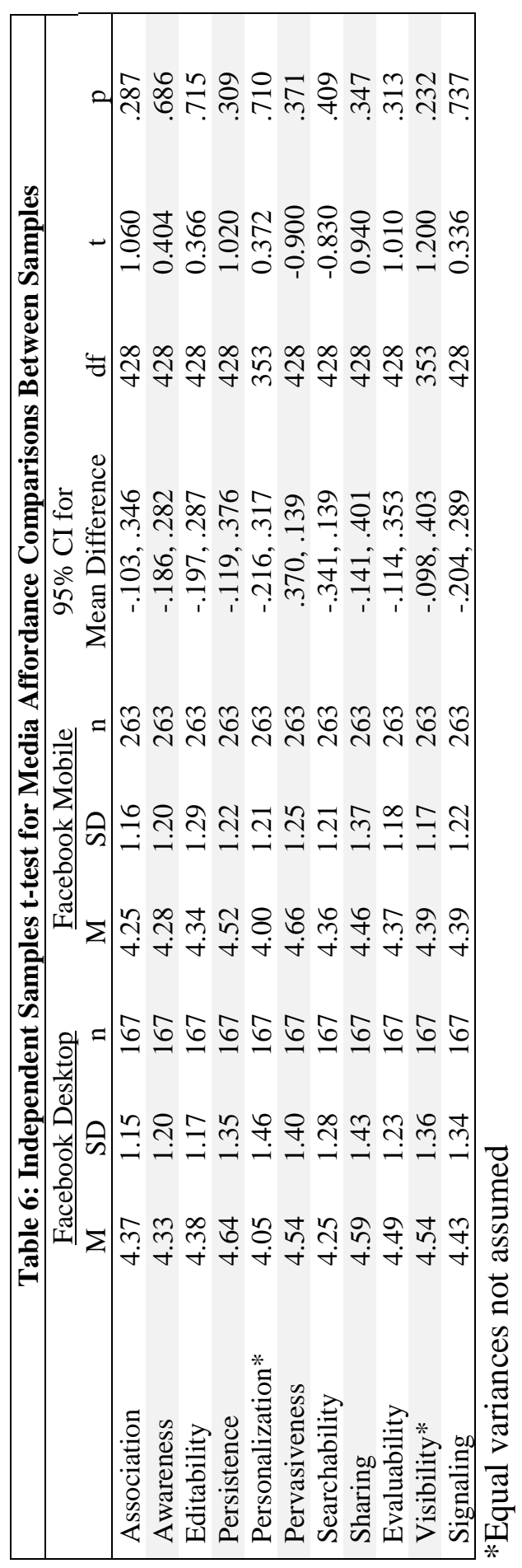




\section{CHAPTER 5: DISCUSSION}

Research continues to show that emotional, interpersonal communication - like giving and receiving emotional support - is alive and well on SNSs and SMAs (Coulson, 2005; Gandy-Guedes et al., 2016; Shaw \& Gant, 2002). This study chose to dig deeper into SNS and SMA communication, as it has become a rich landscape within which to do research on how people provide emotional support to each other via these technologies. In addition, by looking at how perceptions of media affordances are more likely to increase or decrease a user's stress, this study hopes to contribute to achieving a nuanced view of how the Facebook desktop site and Facebook mobile application affect their users. To accomplish this, this study focused on the relationship between perceived emotional support and stress, and media affordances and stress. The results were not all in line with what the literature would suggest or what was expected - some results did support the existing literature, and some did not. The results will be explained and interpreted in the following paragraphs.

For the first few research questions, I was interested in the relationship between stress and emotional support on the Facebook desktop site and Facebook mobile application. First, I found that users of the Facebook mobile application reported greater life stress than users of the Facebook desktop site. However, I found no difference in reported perceived emotional support between the site and the application. Additionally, when testing for a correlation between general life stress and perceived emotional support, I found no correlation between the two for the users of the Facebook desktop site or the Facebook mobile application. Recall that the initial sorting question asked which 
platform participants used most frequently to reach out for emotional support;

participants who chose to answer questions about Facebook's mobile application may use their phones more than desktop computers generally, which in turn might exacerbate their stress, given that mobile phone use has been shown to increase feelings of stress (Augner \& Hacker, 2012). In theory, participants would be using the Facebook mobile application to receive emotional support and thus decrease stress, but there may be an additional component that is adding to their stress instead, either within the Facebook mobile application or as a result of using their phone in general. Considering that no correlation was found between higher perceptions of emotional support and either higher or lower reported general life stress for either group, it could point to the stressors of using a mobile phone over a desktop computer. Finding a correlation between the two could have indicated that perceiving higher levels of emotional support was correlated with lower general life stress, which would have supported the buffering model of stress (Cohen \& Wills, 1985). Not finding a correlation may indicate that emotional support is just a piece of the puzzle, and that other types of support - esteem support and network support (Cobb, 1976), and informational support and tangible support (Cutrona \& Suhr, 1992) may all work together in order to buffer someone from their stress. It would be interesting to study how communication on the Facebook desktop site or Facebook mobile application may be able to provide the other types of support as well. Future research should further study how mobile phone use in general can exacerbate stress.

The next two research questions dug into the concept of frequency of reaching out for emotional support when stressed, and if there was a correlation between perceptions 
of emotional support on the chosen platform and reaching out for emotional support, either by private message or public post. I found what was at first a surprising result: on the Facebook desktop site, the higher the perception of emotional support, the less frequently participants reached out, both by private message and by public post. Additionally, on the Facebook mobile application, the higher the perception of emotional support, the less frequently participants reached out by public post. Though no direction was predicted in the research question, I suggested in the literature review that participants might reach out more frequently they perceived greater emotional support to be available. However, finding the opposite to be true, this seems to indicate that when high levels of emotional support are perceived, there is a lowered need to reach out as frequently. The inverse is true as well: if there is a lower perceived availability of emotional support available on the platform, the user may need to reach out more frequently in order to get their needs fulfilled. These results could even be argued to support the buffering model of stress, which posits that the perception of emotional support being available is more important than the actual utilization of it. This model argues that when people perceive that they have access to emotional support, they are buffered from the harmful effects of stress, but it could also be argued that when people perceive they have access to emotional support, they may not end up reaching out, and just feel better knowing that it is there - and if people perceive that they don't, they may reach out more, seeking it.

Interestingly, I found no correlation between perception of emotional support and reaching out by private message on the Facebook mobile application. I did touch on the 
separation of the main Facebook application and the Facebook messenger application in my literature review, pointing out that users may be affected by this separation on the mobile application, whereas on the desktop site, the messenger function is integrated. Finding that no matter the level of emotional support perceived to be available on the Facebook mobile application, participants reached out no more or less frequently by private message, could support my argument that the messaging function on the mobile application is perceived as far more separated from the "Facebook experience" than on the Facebook desktop site. This is a clear area for future research to consider and dig deeper into how users of the mobile application are affected by having a separate messenger application, and users of the desktop site are affected by having an integrated one.

Finally, the last few research questions explored the concept of studying media through affordances. Recall that I looked first at how each affordance varies from the other affordances within the same sample. I found that when looking at all the responses within the Facebook desktop site sample, persistence, pervasiveness, personalization, and searchability stood out as varying the most from the others. Persistence stood out as having a significantly higher mean than six of the ten other media affordances: editability, searchability, association, personalization, signaling, and awareness. Persistence is defined by Rice et al. (2017) as content being accessible in the same form as it was when originally posted or shared. As an example, on the Facebook desktop site, this would include being able to post a status and return to it later to view it and see any likes or comments it had received. A user of the Facebook desktop site could feel a 
decrease in stress after making a post requesting emotional support - for example, venting about a bad day at work, or mourning the loss of a pet - knowing that they can return to the post and take in the supportive comments from their friends later in the day, and be able to revisit those words of comfort and support whenever they visit the site.

I also found that pervasiveness had a significantly higher mean than four of the ten other media affordances. This is not a majority, but is contextually relevant because of what pervasiveness means. Pervasiveness, as defined by Rice et al. (2017), is the ability to get responses from other users while physically traveling from one location to the next. The Facebook desktop site is generally accessed by a computer, and given the prevalence of desktop computers at home, work, and other public places such as libraries, participants may have perceived the desktop site to be easily accessible in a variety of places, thus enhancing the perception the site is "pervasive". For example, if a user sends a message to a friend requesting advice before they leave work, they may perceive that they can get a response as soon as they get home, which could decrease their stress as soon as they click "post". A site traditionally accessed by a computer would not traditionally be thought of as pervasive, but users may perceive it to be. Again, pervasiveness did not have a significantly higher mean score than a majority of the other media affordances, only four of the ten other media affordances, but future researchers may be interested in studying how an SNS could potentially be perceived as pervasive.

Inversely, there were two media affordances that had significantly lower mean scores than a majority of the other media affordances: personalization and searchability. Personalization, as defined by Rice et al. (2017), is the ability to include content on social 
media that represents the user's personal identity. Personalization had a significantly lower mean than all ten of the other affordances. Searchability is the ability to search for content on a communication technology (Rice et al., 2017), and had a significantly lower mean than six of the other media affordances: evaluability, persistence, pervasiveness, sharing, signaling, and visibility. These two findings are fascinating considering that personalization and searchability are major features of most SNSs and SMAs.

Furthermore, these media affordances, when perceived to exist within the same technology, could suggest that users may worry their emotional support posts could be searched for and linked back to their off-site persona or identity. This could mean that these media affordances may increase stress when imagining that others outside their may become aware of their emotional upset, when they would prefer to keep their feelings or problems private or contained to their network. For example, a user may be hesitant to put up information that is personal and searchable because they don't want their family to know certain things about their life, or they worry about future jobs they apply to searching for information about them online. If they are posting about a personal situation, like a breakup or asking their friends for advice, it may increase their stress knowing that other people may be able to search for it in the future.

I also found similar, but not entirely identical, results within the Facebook mobile application sample. I found that the same two media affordances had significantly higher means than a majority of the other media affordances: pervasiveness and persistence. Pervasiveness had a significantly higher mean than all the media affordances except persistence: association, awareness, editability, personalization, searchability, sharing, 
evaluability, visibility, and signaling. As discussed above, pervasiveness is the ability to get responses while physically traveling (Rice et al., 2017). For the Facebook mobile application, this could mean that users feel a decrease in stress knowing that while they are riding the bus, walking from class to class, or riding home in the car, they can continue accessing emotional support on the application if they need it. Persistence, also discussed above, is the ability to return to content after it has been posted or shared. This was found to have a significantly higher mean than six of the ten other affordances: association, awareness, editability, personalization, searchability, and evaluability. Similar to the desktop site sample, the mobile application sample could perceive the ability to post about their problems, and have people see it long after they have posted it, to decrease their stress.

I found that two of the media affordances had significantly lower means than a majority of the other media affordances: personalization and association. Personalization had a significantly lower mean than all ten other media affordances: association, awareness, editability, evaluability, persistence, pervasiveness, searchability, sharing, signaling, and visibility. This is the same finding as above, for the Facebook desktop site, and potentially carries the same fascinating implications. Association had a significantly lower mean than seven of the ten other affordances: evaluability, persistence, pervasiveness, searchability, sharing, signaling, and visibility. Association, as defined by Rice et al. (2017) is the ability to establish connections between users and other users, or users and content. On the Facebook mobile application, association is found within lists of a user's friends (who they are connected with) or pages that they've liked. Facebook 
also has a feature where posts that a user likes are shown to their friends, with a qualifier saying that that user liked it. It seems that one or more of these features could be negatively impacting users' stress. For example, a user may worry that they cannot like a page or connect with certain friends without those associations being connected back to them in the future.

In research, it is just as important when no significant findings are discovered, as it still gives valuable information about the participants under study. In this case, when comparing the media affordance mean scores between the two samples, no significant differences were found. As stated, this measure looked into how perceptions of these affordances affected stress, and these results suggest that these media affordances do not affect stress differently when using the Facebook desktop site or Facebook mobile application. While there are differences between the two that were covered in the literature review, this result is not entirely unexpected. The desktop site and mobile application still provide users access to the same group of people, and provide similar or identical functions, even if the design or access (by computer or mobile phone) are different. As suggested by this result, using the desktop site or mobile application to access Facebook does not seem to affect stress in terms of the affordances offered. However, this does not mean that they don't affect participants in different ways - after all, I also found that participants reached out less frequently by private message when they perceived a higher level of emotional support to be available on the Facebook desktop site, but found no correlation for the Facebook mobile application. I also found a difference between participants in each sample of general life stress. This result for a lack 
of differences in how media affordances affect stress between the platforms does not rule out differences between how the two platforms are perceived, or how they affect their users differently, only that future research should dig more deeply into it.

As seen above, there were similarities and differences between the two samples.

For example, persistence was found to be more likely to decrease stress on both platforms, whereas association was found to be less likely to decrease stress only on the Facebook mobile application. This does suggest that looking at media affordances, rather than the specific features of the technology itself, can help us better understand communication technologies in the long term.

\section{Limitations to Present Study}

There are a few limitations to this study that are worth discussing. First, the media affordances portion of this study was entirely exploratory, and while the lead researcher worked with other experts who research media affordances, applying it in a stress-based way to SNSs and SMAs is entirely new territory. Hopefully it will provide a basis for future research to build on, but did have a lack of previous research to rest on. In addition, this was an entirely quantitative study, with no open-ended questions or qualitative methods. While each method has its own weaknesses, a weakness of quantitative survey data is potential errors in self-report data. Participants, for example, may not be as aware of how media affordances available on a site affect their stress, and this could be an excellent avenue for future research to explore. Additionally, the survey was quite long, and there is always the possibility of survey fatigue. There were four measures in total, although this thesis only looked at the first three measures, which 
hopefully minimizes any survey fatigue that participants may have been feeling by the end. Finally, this was a self-report measure, which relies on the honesty and memory of the participants.

If I could go back and change anything, it would be the initial sorting question. The biggest benefit to this study was that we recruited participants to form a random, nationally representative sample as best we could - however, we also had to ensure that participants were answering questions about a media they were familiar with. Our sorting question asked participants which media they used most frequently to reach out for emotional support when stressed, and sorted them into the corresponding survey of the option they chose. Instead of this, I would allow participants to choose any platform they use to reach out for emotional support when stressed, with perhaps a minimum of using it once per week, and randomly sort them into any version they chose. I think this would add back in an element of randomness that was slightly lost.

\section{Conclusion}

In all, there were some valuable findings in this study relating to media affordances, emotional support, stress, and the differences between the Facebook desktop site and Facebook mobile application. While this study may not have seen as many differences as prompted by research and explicated in the literature review, some differences were found, including a significantly higher amount of life stress for Facebook mobile application users over Facebook desktop application users. There were also findings that suggest that users are able to distinguish which media affordances are more likely to decrease their stress, which is an important contribution to the study of 
media affordances. Although there were no significant differences when comparing the Facebook desktop site to the Facebook mobile application, comparing SNSs and SMAs from different companies could yield differences (i.e. the Facebook desktop site versus Instagram), and is an area that should be considered by researchers in the future. Just as researchers have worked towards a better understanding of how internet use affects its users, researchers should continue to work towards finding a way to research SNSs and SMAs that contributes to a larger foundation of literature. Through researching how media affordances of SNSs and SMAs affect interpersonal phenomena like perceived emotional support, stress, and other important communication-based processes, a more long-lasting understanding can be achieved of how these media affect their users. 


\section{REFERENCES}

Afifi, T. D., Zamanzadeh, N.,_Harrison, K., \& Acevedo Callejas, M. (2018). WIRED: The impact of media and technology use on stress (cortisol) and inflammation (interleukin IL-6) in fast paced families. Computers in Human Behavior, 81, 265273. https://doi.org/10.1016/j.chb.2017.12.010

Albrecht, T. L., \& Adelman, M. B. (1984). Social support and life stress. Human Communication Research, 11(1), 3-32.

Augner, C., \& Hacker, G. W. (2012). Associations between problematic mobile phone use and psychological parameters in young adults. International journal of public health, 57(2), 437-441.

Beckley, M. (2006). Community Participation Following Cerebrovascular Accident: Impact of the Buffering Model of Social Support. Retrieved September 8, 2018, from

http://link.galegroup.com.proxy.lib.pdx.edu/apps/doc//PPNU?sid=googlescholar

Bolger, N., Zuckerman, A., \& Kessler, R. C. (2000). Invisible support and adjustment to stress. Journal of Personality and Social Psychology, 79, 953-961.

boyd, d. b. \& Ellison, N. B. (2007). Social network sites: Definition, history, and scholarship. Journal of Computer-Mediated Communication, 13(1), 210-230. doi: 10.1111/j.10836101.2007.00393.x

Burleson, B. R. (2003). The experience and effects of emotional support: What the study Of cultural and gender differences can tell us about close relationships, emotion, And interpersonal communication. Personal Relationships, 10(1), 1-23. 
https://doi.org/10.1111/1475-6811.00033

Burleson, B. R. (1984). Comforting communication. In H. E. Sypher \& J. L. Applegate (Eds.), Communication by children and adults: Social cognitive and strategic processes (pp. 63-104). Beverly Hills, CA: Sage.

Chung, J. E. (2014). Social Networking in Online Support Groups for Health: How Online Social Networking Benefits Patients. Journal Of Health Communication, 19(6), 639-659. doi:10.1080/10810730.2012.757396

Cobb, S. (1976). Social Support as a Moderator of Life Stress. Psychosomatic Medicine, 38(5), 300-314.

Cohen, S., Kamarck, T., \& Mermelstein, R. (1983). A global measure of perceived stress. Journal of Health and Social Behavior, 24(4), 385-396.

Cohen, S., Kessler, R. C., \& Gordon, L. U. (1997). Measuring Stress: A Guide for Health and Social Scientists. Oxford University Press.

Cohen, S., \& Wills, T. A. (1985). Stress, social support, and the buffering hypothesis. Psychological Bulletin, 98(2), 310-357. https://doi.org/http://dx.doi.org/10.1037/0033-2909.98.2.310

Constine, J. (2017, September 14). Facebook Messenger Day hits 70M daily users as the app reaches 1.3B monthlies. Retrieved May 6, 2018, from http://social.techcrunch.com/2017/09/14/facebook-messenger-1-3-billion/

Cutrona, C. E., \& Suhr, J. A. (1992). Controllability of stressful events and satisfaction with spouse support behaviors. Communication Research, 19(2), 154-174. https://doi.org/10.1177/009365092019002002 
Coulson, N. S. (2005). Receiving Social Support Online: An Analysis of a ComputerMediated Support Group for Individuals Living with Irritable Bowel Syndrome. CyberPsychology \& Behavior, 8(6), 580-584. https://doi.org/10.1089/cpb.2005.8.580

Eaton, W. W. (1978). Life Events, Social Supports, and Psychiatric Symptoms: A ReAnalysis of the New Haven Data. Journal of Health and Social Behavior, 19(2), 230-234. https://doi.org/10.2307/2136537

Ellison, N. B. \& boyd, d. (2013). Sociality through Social Network Sites. In Dutton, W. H. (Ed.), The Oxford Handbook of Internet Studies. Oxford: Oxford University Press, pp. 151-172.

Ellison, N. B., Steinfield, C., \& Lampe, C. (2007). The Benefits of Facebook "Friends:" Social Capital and College Students' Use of Online Social Network Sites. Journal of Computer-Mediated Communication, 12(4), 1143-1168. https://doi.org/10.1111/j.1083-6101.2007.00367.x

Ellison, N. B., \& Vitak, J. (2015). Social media affordances and their relationship to social capital processes. In S. Sundar (Ed.), The handbook of psychology of communication technology (pp. 205-227). Boston: Wiley-Blackwell.

Evans, S. K., Pearce, K. E., Vitak, J., \& Treem, J. W. (2016). Explicating affordances: A conceptual framework for understanding affordances in communication research. Journal of Computer-Mediated Communication, 22(1), 35-52.

Gandy-Guedes, M. E., Vance, M. M., Bridgewater, E. A., Montgomery, T., \& Taylor, K. (2016). Using Facebook as a tool for informal peer support: a case example. 
Social Work Education, 35(3), 323-332.

https://doi.org/10.1080/02615479.2016.1154937

Gibson, J. J. (1979). The ecological approach to visual perception. Hillsboro, NJ: Erlbaum.

Graham, J. M., \& Barnow, Z. B. (2013). Stress and social support in gay, lesbian, and heterosexual couples: Direct effects and buffering models. Journal of Family Psychology, 27(4), 569-578. https://doi.org/http://dx.doi.org.proxy.lib.pdx.edu/10.1037/a0033420

Greene, J. A., Choudhry, N. K., Kilabuk, E., \& Shrank, W. H. (2011). Online Social Networking by Patients with Diabetes: A Qualitative Evaluation of Communication with Facebook. Journal of General Internal Medicine, 26(3), 287-292. https://doi.org/10.1007/s11606-010-1526-3

Greenwood, S., Perrin, Andrew, \& Duggan, M. (2016, November 11). Social Media Update 2016. Retrieved October 3, 2017, from http://www.pewinternet.org/2016/11/11/social-media-update-2016/

Guan, S.-S. A., Chiang, J. J., Sherman, L. E., Nguyen, J., Tsui, Y., \& Robles, T. F. (2017). Culture moderates the effect of social support across communication contexts in young adult women in the United States. Computers in Human Behavior, 75, 775-784. https://doi.org/10.1016/j.chb.2017.05.048

Kaplan, B. H., Cassel, J. C., \& Gore, S. (1977). Social Support and Health. Medical Care, $15(5), 47-58$. 
Kaplan, A. M., \& Haenlein, M. (2010). Users of the world, unite! The challenges and opportunities of Social Media. Business Horizons, 53(1), 59-68. https://doi.org/10.1016/j.bushor.2009.09.003

Kraut, R., Patterson, M., Lundmark, V., Kiesler, S., Mukophadhyay, T., \& Scherlis, W. (1998). Internet paradox: a social technology that reduces social involvement and psychological well-being. American Psychologist, 53, 1017-1031.

Liu, H. (2007). Social Network Profiles as Taste Performances. Journal of ComputerMediated Communication, 13(1), 252-275. https://doi.org/10.1111/j.10836101.2007.00395.x

Liu, C.-Y., \& Yu, C.-P. (2013). Can Facebook Use Induce Well-Being? CyberPsychology, Behavior \& Social Networking, 16(9), 674-678. https://doi.org/10.1089/cyber.2012.0301

McKinley, C._(2013). Applying a distress-deterring approach to examine how emotional support predicts perceived stress and stress-related coping response. Southern Communication Journal, 78(5), 387-404. doi:10.1080/1041794X.2013.839737

Meier, A. (2002). An Online Stress Management Support Group for Social Workers. Journal of Technology in Human Services, 20(1-2), 107-132. https://doi.org/10.1300/J017v20n01_10

Myers, J. K., Lindenthal, J. J., \& Pepper, M. P. (1975). Life Events, Social Integration and Psychiatric Symptomatology. Journal of Health and Social Behavior, 16(4), 421-427. https://doi.org/10.2307/2136613

Norman, D. The Design of Everyday Things Revised and Expanded Edition. (2013). New 
York: Basic Books.

Rice, R. E., Evans, S. K., Pearce, K. E., Sivunen, A., Vitak, J. and Treem, J. W. (2017), Organizational media affordances: Operationalization and associations with media use. Journal of Communication, 67, 106-130. doi:10.1111/jcom.12273

Shaw, L. H., \& Gant, L. M. (2002). In Defense of the Internet: The Relationship between Internet Communication and Depression, Loneliness, Self-Esteem, and Perceived Social Support. CyberPsychology \& Behavior, 5(2), 157-171. https://doi.org/10.1089/109493102753770552

Shensa, A., Sidani, J. E., Lin, L. yi, Bowman, N. D., \& Primack, B. A. (2016). Social Media Use and Perceived Emotional Support Among US Young Adults. Journal of Community Health, 41(3), 541-549. https://doi.org/10.1007/s10900-015-0128-8

Thoits, P. A. (1982). Conceptual, Methodological, and Theoretical Problems in Studying Social Support as a Buffer Against Life Stress. Journal of Health and Social Behavior, 23(2), 145-159. https://doi.org/10.2307/2136511

Weber, K. D., \& Patterson, B. R. (1996). Construction and Validation of a Communication Based Emotional Support Scale. Communication Research Reports, 13(1), 68-76.

Welbourne, J. L., Blanchard, A. L., \& Wadsworth, M. B. (2013). Motivations in virtual health communities and their relationship to community, connectedness and stress. Computers in Human Behavior, 29(1), 129-139.

https://doi.org/10.1016/j.chb.2012.07.024 
Wright, K. B. (2002). Social support within an on-line cancer community: an assessment of emotional support, perceptions of advantages and disadvantages, and motives for using the community from a communication perspective. Journal of Applied Communication Research, 30(3), 195-209. https://doi.org/10.1080/00909880216586

Wright, K. B. (2012). Emotional Support and Perceived Stress Among College Students Using Facebook.com: An Exploration of the Relationship Between Source Perceptions and Emotional Support. Communication Research Reports, 29(3), 175-184. https://doi.org/10.1080/08824096.2012.695957 
Appendix A: Informed Consent Form

\section{Consent Form}

\section{Social Media Survey}

You are being invited to participate in a research study about how using social media or interacting face-to-face with another person or other people affects a person's stress. This study is being conducted by Dr. Erin Spottswood from the Communication Department at Portland State University.

There are no known risks if you decide to participate in this research study. There are no costs to you for participating in the study. The information you provide will be used to further uncover how and why people respond or do not respond to posts they see on Facebook. The questionnaire will take about 15-20 minutes to complete. The information collected may not benefit you directly, but the information learned in this study should provide more general benefits.

This survey is anonymous, however, absolute anonymity cannot be guaranteed over the Internet. However, no one will be able to identify you or your answers, and no one will know whether or not you participated in the study. Individuals from the Institutional Review Board may inspect these records. Should the data be published, no individual information will be disclosed.

Your participation in this study is voluntary. By completing the online survey, you are voluntarily agreeing to participate. You are free to decline to answer any particular question you do not wish to answer for any reason.

If you have any questions about the study, please contact Dr. Spottswood at University Center Building 520 SW Harrison Street, Suite 440, Portland, OR 97201, 503.725.5810.

The Portland State University Institutional Review Board has reviewed this project. If you have any concerns about your rights in this study, please contact the PSU Office of Research Integrity at (503) 725-2227 or email hsrrc@pdx.edu.

By clicking "accept" at the end of this form, you are consenting to participate in this survey.

IF you do not consent, please click "decline" to navigate away from the survey.

I accept

I decline to accept 
Appendix B: Use of Communication Channels for Support when you are Stressed

When you are feeling stressed, how often do you reach out to others in the following ways?

1 Face to face one-to-one conversations

2 Face to face conversations with more than one person, a group of people

\section{Other Media}

3 Sending/receiving emails

4 Telephone (landline or mobile) calls

5 Short messages (including text messages, Google Chat, other chat programs)

6 Video calls (Google hangouts, Facetime, other video communication)

7 Public Facebook desktop site post

8 Public Message via the Facebook desktop site

9 Public Facebook mobile application post

10 Private Message via the Facebook mobile application

11 Public Instagram post

12 Private Message via Instagram

13 Public Reddit post

14 Private message via Reddit

15 Public Snapchat post

16 Private message via Snapchat

Scale: 1 Never, 2 a few times a year or less, 3 once a month or less, 4 a few times a month, 5 once a week, 6 a few times a week, 7 every day, 8 a few times a day, 9 many times a day 
Appendix C: Perceived Stress Scale

For the following questions please indicate how often you felt or thought a certain way.

1. In the last month, how often have you been upset because of something that happened unexpectedly?

2. In the last month, how often have you felt that you were unable to control the important things in your life?

3. In the last month, how often have you felt nervous and "stressed"?

4. In the last month, how often have you felt confident about your ability to handle your personal problems?

5. In the last month, how often have you felt that things were going your way?

6. In the last month, how often have you found that you could not cope with all the things that you had to do?

7. In the last month, how often have you been able to control irritations in your life?

8. In the last month, how often have you felt that you were on top of things?

9. In the last month, how often have you been angered because of things that were outside of your control?

10. In the last month, how often have you felt difficulties were piling up so high that you could not overcome them?

Scale: 0 Never, 1 Almost Never, 2 Sometimes, 3 Fairly Often, 4 Very Often

Adapted from Source:

Cohen, S., Kamarck, T., \& Mermelstein, R. (1983). A global measure of perceived

stress. Journal of Health and Social Behavior, 24(4), 385-396. Doi: 10.2307/213640 
Appendix D: Media Affordances Possible on the Facebook Desktop Site ${ }^{1}$

The next set of questions will ask you to think about how being able to use the Facebook desktop site in a variety of different ways may increase or decrease your stress.

If you don't believe a particular action is possible on the Facebook desktop site, please choose "Not possible".

If you are unsure how a particular action on Facebook desktop site affects your stress, please choose "Do not know".

[association] [as noted, the affordance label is not included in the survey]

1. When I find information I already knew or was aware of while using the Facebook desktop site, it is more likely to...

2. When I find new information I did not know or wasn't aware of while using the Facebook desktop site, it is more likely to...

3. When I find people I already know or am aware of while using the Facebook desktop site, it is more likely to...

4. When I find new people I did not know or wasn't aware of while using the Facebook desktop site, it is more likely to...

\section{[awareness]}

5. When I become aware of the information others have while using the Facebook desktop site, it is more likely to...

6. When I become aware of activities, opinions, or locations of others while using the Facebook desktop site, it is more likely to...

[awareness]

7. When I keep up-to-date with what others are posting on the Facebook desktop site, it is more likely to...

8. When I keep up-to-date with the Facebook desktop site policies and norms, it is more likely to...

\section{[editability]}

9. When I edit others' content (i.e. deleting comments) after they have posted it on the Facebook desktop site, it is more likely to...

10. When I edit my content (i.e. editing or deleting posts, comments, etc.) on the Facebook desktop site after I have posted it, it is more likely to...

11. When I create or edit a post along with other people on the Facebook desktop site, it is more likely to... 
[persistence]

12. When I maintain relations with others on the Facebook desktop site despite changes in activities or location, it is more likely to...

13. When I have my information or comments stay available after I post them on the

Facebook desktop site, it is more likely to...

\section{[personalization]}

14. When I include information that presents my personal identity on the Facebook desktop site, it is more likely to...

15. When I include photos that present my personal identity on the Facebook desktop site, it is more likely to...

16. When I include other content that presents my personal identity on the Facebook desktop site, it is more likely to...

\section{[pervasiveness]}

17. When I get responses to my requests from others quickly while using the Facebook desktop site, it is more likely to...

18. When I communicate with others on the Facebook desktop site while moving, commuting, or traveling, it is more likely to...

\section{[searchability]}

19. When I search for information or people by entering search words on the Facebook desktop site, it is more likely to...

20. When I search for information or people by following links between content on the Facebook desktop site, it is more likely to...

21. When I search for tags or keywords that someone else has added to content on the Facebook desktop site, it is more likely to...

\section{[sharing]}

22. When I create groups for sharing information about thoughts, feelings, concerns, ideas, etc. on the Facebook desktop site, it is more likely to...

23. When I share my posts, updates, photos, videos, and other types of content with other people on the Facebook desktop site, it is more likely to ...

\section{[evaluatability]}

24. When I see other people's evaluation of information through their recommendations on the Facebook desktop site, it is more likely to...

25. When I see other people's evaluation of information through their comments on the Facebook desktop site, it is more likely to...

26. When I see other people's evaluation of information through their liking on the Facebook desktop site, it is more likely to... 
27. When I see other people's evaluation of information through their tagging on the Facebook desktop site, it is more likely to...

\section{[visibility]}

28. When I see other people's answers to other people's questions on the Facebook desktop site, it is more likely to...

29. When I see interactions or links with other people on the Facebook desktop site, it is more likely to...

30. When I see the number of others who have "liked" or linked to the same content on the Facebook desktop site, it is more likely to...

\section{[signaling]}

31. When I receive notifications about other information or updates that are similar to what I have just been looking at on the Facebook desktop site, it is more likely to... 32. When I receive notifications about other people's information or updates on the Facebook desktop site, it is more likely to....

\section{Scale:}

... (1) Strongly increase my stress, (2) increase my stress, (3) increase my stress a little bit, (4) not increase or decrease my stress, (5) decrease my stress a little bit, (6) decrease my stress, (7) strongly decrease my stress, (8) not possible, (9) [do not know] - so, need to recode 9 as "do not know" and thus as missing

\section{Adapted from Source:}

Rice, R. E., Evans, S. K., Pearce, K. E., Sivunen, A., Vitak, J., \& Treem, J. W. (2017). Organizational media affordances: Operationalization and associations with media use. Journal of Communication, 67(1), 106-130. 
Appendix E: Communication Based Emotional Support Scale (CBESS)

The following questions are concerned with how your Facebook desktop site friends communicate with you on a variety of different issues. For each statement, please respond by clicking on number that best represents your agreement with that statement.

1. There are people on the Facebook desktop site that help me work through my thoughts and feelings about major life decisions (eg. career choice)

$$
\begin{aligned}
& 5 \text { - Almost Always True } \\
& 4 \text { - Often True } \\
& 3 \text { - Occasionally True } \\
& 2 \text { - Rarely True } \\
& 1 \text { - Almost Never True }
\end{aligned}
$$

2. There are people on the Facebook desktop site that will do things that they know will upset me [r]

$$
\begin{aligned}
& 5 \text { - Almost Always True } \\
& 4 \text { - Often True } \\
& 3 \text { - Occasionally True } \\
& 2 \text { - Rarely True } \\
& 1 \text { - Almost Never True }
\end{aligned}
$$

3. There are people on the Facebook desktop site that patiently and sensitively listen to me "let off steam" about an outside problem that I am having

5 - Almost Always True

4 - Often True

3 - Occasionally True

2 - Rarely True

1 - Almost Never True

4. When I tell people on the Facebook desktop site about a problem that I am having, they don't seem to be paying attention $[\mathrm{r}]$

$$
\begin{aligned}
& 5 \text { - Almost Always True } \\
& 4 \text { - Often True } \\
& 3 \text { - Occasionally True } \\
& 2 \text { - Rarely True } \\
& 1 \text { - Almost Never True }
\end{aligned}
$$

5. There are people on the Facebook desktop site that help me cope with problems concerning other friends and/or family members

$$
\begin{aligned}
& 5 \text { - Almost Always True } \\
& 4 \text { - Often True } \\
& 3 \text { - Occasionally True } \\
& 2 \text { - Rarely True } \\
& 1 \text { - Almost Never True }
\end{aligned}
$$

6. People on the Facebook desktop site avoid me when I am depressed [r]

5 - Almost Always True 


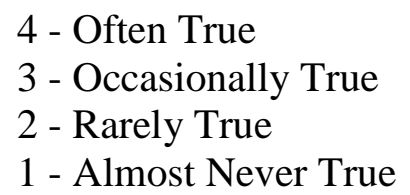

7. There are people on the Facebook desktop site messenger that are good listeners when I am upset

$$
\begin{aligned}
& 5 \text { - Almost Always True } \\
& 4 \text { - Often True } \\
& 3 \text { - Occasionally True } \\
& 2 \text { - Rarely True } \\
& 1 \text { - Almost Never True }
\end{aligned}
$$

8. When I tell people on the Facebook desktop site about a problem that I am having, they respond with "If you think that is bad, listen to this..." [r]

$$
\begin{aligned}
& 5 \text { - Almost Always True } \\
& 4 \text { - Often True } \\
& 3 \text { - Occasionally True } \\
& 2 \text { - Rarely True } \\
& 1 \text { - Almost Never True }
\end{aligned}
$$

9. There are people on the Facebook desktop site that never listen to my problems [r]

5 - Almost Always True

4 - Often True

3 - Occasionally True

2 - Rarely True

1 - Almost Never True

10. There are people on the Facebook desktop site that say and do supportive things for me when I am feeling down

$$
\begin{aligned}
& 5 \text { - Almost Always True } \\
& 4 \text { - Often True } \\
& 3 \text { - Occasionally True } \\
& 2 \text { - Rarely True } \\
& 1 \text { - Almost Never True }
\end{aligned}
$$

11. When I want to talk to people on the Facebook desktop site about what is bothering me, they seem to have something else to do [r]

$$
\begin{aligned}
& 5 \text { - Almost Always True } \\
& 4 \text { - Often True } \\
& 3 \text { - Occasionally True } \\
& 2 \text { - Rarely True } \\
& 1 \text { - Almost Never True }
\end{aligned}
$$

12. There are people on the Facebook desktop site that show genuine concern for my problems

$$
\begin{aligned}
& 5 \text { - Almost Always True } \\
& 4 \text { - Often True } \\
& 3 \text { - Occasionally True }
\end{aligned}
$$


2 - Rarely True

1 - Almost Never True

13. When I talk to people on the Facebook desktop site about a problem that I have, they tell me that I am overreacting [r]

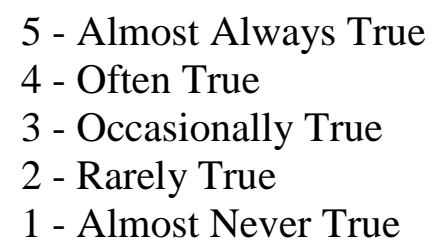

14. There are people on the Facebook desktop site that give me good advice when I ask for it

5 - Almost Always True

4 - Often True

3 - Occasionally True

2 - Rarely True

1 - Almost Never True

15. I don't like to talk about things that are bothering me with people on the

Facebook desktop site because they will think that I am mad at them and get defensive [r]
5 - Almost Always True
4 - Often True
3 - Occasionally True
2 - Rarely True
1 - Almost Never True

16. There are people on the Facebook desktop site that make it very easy to discuss my personal feelings
5 - Almost Always True
4 - Often True
3 - Occasionally True
2 - Rarely True
1 - Almost Never True

17. There are people on the Facebook desktop site that tell me what I should do even when I don't ask for advice [r]

5 - Almost Always True

4 - Often True

3 - Occasionally True

2 - Rarely True

1 - Almost Never True

18. There are people on the Facebook desktop site that listen to my side of the story even if they think that I am wrong

5 - Almost Always True

4 - Often True

3 - Occasionally True 
2 - Rarely True

1 - Almost Never True

19. There are people on the Facebook desktop site that don't understand that when I am in a bad mood, sometimes I just need to "blow off steam" [r]

5 - Almost Always True

4 - Often True

3 - Occasionally True

2 - Rarely True

1 - Almost Never True

20. There are people on the Facebook desktop site that make an effort to make me feel better when I am depressed

5 - Almost Always True

4 - Often True

3 - Occasionally True

2 - Rarely True

1 - Almost Never True

Adapted from Source:

Weber, K. D., \& Patterson, B. R. (1996). Construction and Validation of a Communication Based Emotional Support Scale. Communication Research Reports, 13(1), 68-76. 
Appendix F: Demographics

1) What is your current job status? Please pick the category that best describes your current job status.
a) Working full time
b) Working part time
c) Graduate student
d) Undergraduate student
e) Home maker
f) Unable to work
g) Unemployed Retired

2) What is your ethnicity? (May select more than one)
a) White/Anglo/Caucasian/Middle Eastern
b) Black/African American
c) Asian
d) American Indian or Alaskan Native
e) Hispanic or of Latino origin
f) Other, please specify:

3) In which year were you born:

[Drop down menu]

4) What is your sex?
a) Female
b) Male
c) Other [open ended text box]

Adapted from source:

Spottswood \& Wohn, under review 